CONFORMAL GEOMETRY AND DYNAMICS

An Electronic Journal of the American Mathematical Society

Volume 8, Pages 87-114 (March 29, 2004)

S $1088-4173(04) 00102-\mathrm{X}$

\title{
LANDING PROPERTY OF STRETCHING RAYS FOR REAL CUBIC POLYNOMIALS
}

\author{
YOHEI KOMORI AND SHIZUO NAKANE
}

\begin{abstract}
The landing property of the stretching rays in the parameter space of bimodal real cubic polynomials is completely determined. Define the Böttcher vector by the difference of escaping two critical points in the logarithmic Böttcher coordinate. It is a stretching invariant in the real shift locus. We show that stretching rays with non-integral Böttcher vectors have non-trivial accumulation sets on the locus where a parabolic fixed point with multiplier one exists.
\end{abstract}

\section{Contents}

1. Introduction

2. Complex cubic polynomials

2.1. The parameter space of complex cubic polynomials

2.2. The mappings $h_{P}$ and $\varphi_{P}$

2.3. The connectedness locus and the escape locus

2.4. The wring construction and stretching rays

2.5. Invariants on stretching rays

3. Real cubic polynomials

3.1. The parameter space of real cubic polynomials $\quad 93$

3.2. Critical orbits of $P \in \mathbb{R}_{+}^{2} \backslash \mathcal{C}(\mathbb{R})$

3.3. Stretching rays in $\mathbb{R}_{+}^{2}$

3.4. Böttcher vectors in the real shift locus

4. Landing property of stretching rays in $\mathcal{E}_{2}(\mathbb{R}) \cap \mathcal{R}_{2} \quad 96$

5. Landing property of stretching rays in $\mathcal{E}_{1}(\mathbb{R}) \quad 98$

5.1. Hyperbolic rays land $\quad 98$

5.2. Hyperbolic parameters are landing points $\quad 98$

5.3. Non-hyperbolic rays also land 99

5.4. Non-hyperbolic parameters are also landing points 100

6. Landing and non-landing of stretching rays in $\mathcal{R}_{0} \quad 101$

6.1. Stretching rays with integral Böttcher vectors $\quad 102$

6.2. Parametrization of $\operatorname{Per}_{1}(1)$ by Fatou vectors 103

6.3. Stretching rays with irrational Böttcher vectors $\quad 105$

6.4. Stretching rays with rational Böttcher vectors 108

7. Accumulation sets of stretching rays in $\mathcal{R}_{0} \quad 111$

References

Received by the editors May 15, 2003 and, in revised form, November 20, 2003.

2000 Mathematics Subject Classification. Primary 37F45; Secondary 37F30.

Key words and phrases. Stretching rays, parabolic implosion, radial Julia set. 


\section{INTRODUCTION}

It is well known that the behaviour of the critical points plays an important role in the dynamics of polynomials. The dynamical positions of the critical points or critical values often yield a parametrization of the parameter space. For example, in case of quadratic polynomials, there is only one critical point and the Böttcher coordinate of the critical value gives the parametrization of the complement of the Mandelbrot set, the connectedness locus. Compared with the quadratic polynomials, the parameter space of cubic polynomials is fairly complicated since they have two critical points. In fact, the connectedness locus for the cubic polynomials is not locally connected (cf. La ), while the Mandelbrot set is conjectured to be locally connected. This is characteristic to cubic or higher degree polynomials; the relative dynamical position of the two critical points plays an essential role in its proof.

Stretching rays are defined through a qc-deformation of almost complex structures, called the stretching, along the radial directions of the Böttcher coordinates. In the quadratic case, this corresponds to the stretching of the radial Böttcher coordinate of the critical value and gives rise to external rays. Thus the notion of stretching rays is a generalization of that of external rays for the Mandelbrot set to higher degree polynomials.

In the quadratic case, the external angle, the angular Böttcher coordinate of the critical value, is invariant on each ray, labels each ray and determines a combinatorial property of its dynamics. This enables us to show those rays with rational angles land at some points. However, in the cubic case, they are real one-dimensional rays in the complex two-dimensional parameter space. Hence, it is not easy to find enough invariants to characterize each ray. This makes it difficult to study their landing property in the whole parameter space.

In this article, we investigate the landing property of stretching rays in the family of real cubic polynomials:

$$
P(z)=P_{A, B}(z)=z^{3}-3 A z+\sqrt{B} ; \quad A, B>0 .
$$

Since this family is real two-dimensional, one invariant is enough to characterize each stretching ray. Moreover, the boundary of the connectedness locus is simple and smooth; it consists of parts of two real algebraic curves $\operatorname{Per}_{1}(1)$ and $\operatorname{Preper}_{(1) 1}$ in the notation of [Mi1]. Therefore stretching rays must accumulate on these curves. Figure 1 is the parameter space of our family. The black region is the connectedness locus. Its complement is gradated in order to emphasize stretching rays.

In the real shift locus $\mathcal{E}_{2}(\mathbb{R})$, we will define a stretching-invariant, the Böttcher vector, by the difference of two critical points in the coordinate: $\zeta=\log \log \left|\varphi_{P}(z)\right| /$ $\log 3$, where $\varphi_{P}$ is the Böttcher coordinate for $P$. It follows that stretching rays in the real shift locus are level curves of the Böttcher vector map. On the other hand, if a map in the escape locus has an attracting cycle of period $k$ with multiplier $\lambda$, $k$ and $\lambda$ are both invariant on the stretching ray through the map. Hence we can draw their pictures; see Figures 1 and 2 . There $\mathcal{E}_{2}(\mathbb{R})$ is gradated by the Böttcher vector, while the locus $\mathcal{E}_{1}(\mathbb{R})$, where only one critical point escapes, is gradated by the period of the attracting cycle.

Here we give the summary of our results. Let $\operatorname{Per}_{1}(1)$ be the locus consisting of maps having a parabolic fixed point with multiplier one. Our main result, Theorem 6.1, states that stretching rays with non-integral Böttcher vectors above $\operatorname{Per}_{1}(1)$ do not land at any point on $\operatorname{Per}_{1}(1)$ (but, of course, they accumulate on it). We 


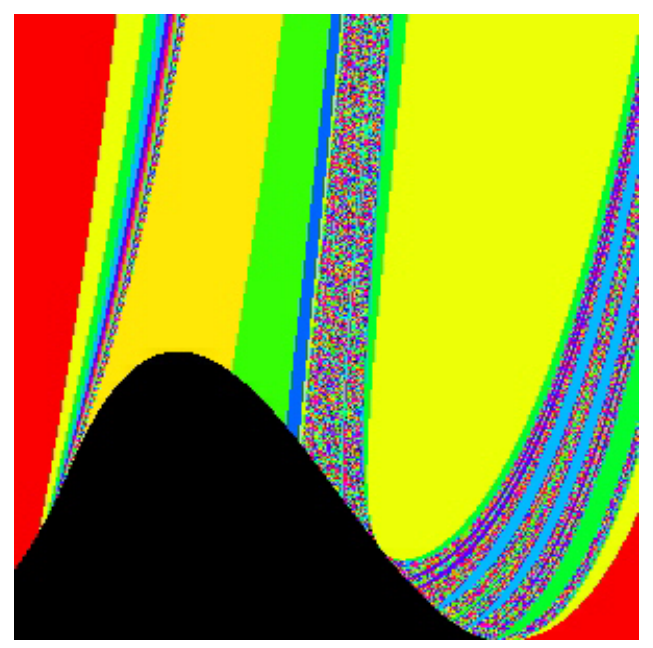

FIGURE 1. The parameter space of real cubic polynomials

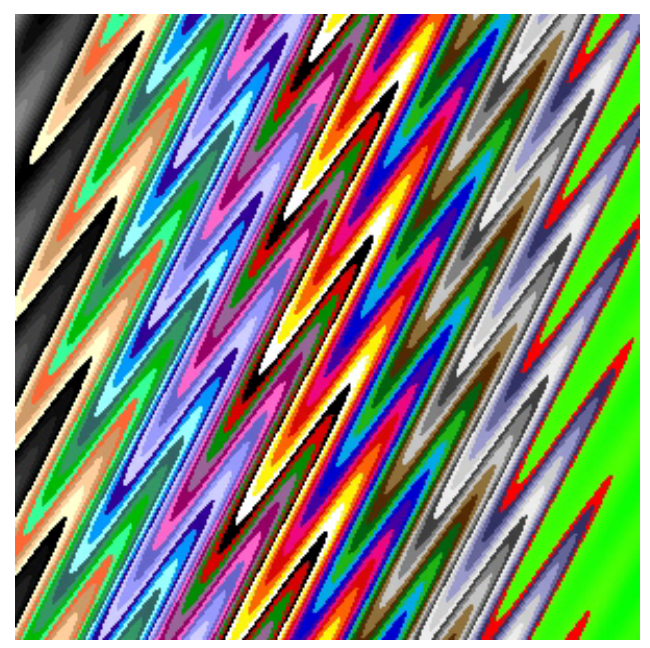

FiguRE 2. An enlargement of Figure 1 Oscillation of stretching rays

also show that each stretching ray with integral Böttcher vector above $\operatorname{Per}_{1}(1)$ does land at a point on $\operatorname{Per}_{1}(1)$. Figure2 2 is an enlargement of a small box near the leftdown corner of Figure 1 and suggests that stretching rays oscillate like the graph of $\sin (1 / x)$ as they approaches $\operatorname{Per}_{1}(1)$. As the Böttcher vector tends to an integer, the width of oscillation tends to zero. This allows the coexistence of landing and non-landing rays.

Historically, the locus $\operatorname{Per}_{1}(1)$ has been investigated to reveal the features of the cubic dynamics different from that of quadratic dynamics. We add our result as one more evidence to it; see [D-H], [Mi1], [La and [E-Y].

Let $\operatorname{Preper}_{(1) 1}$ be the locus consisting of maps whose critical value $P(-\sqrt{A})$ is a fixed point. We show that all stretching rays in the real shift locus below $\operatorname{Per}_{1}(1)$ land on $\operatorname{Preper}_{(1) 1}$ and we characterize the landing points: $P^{k}(\sqrt{A})=-\sqrt{A}$ for 
some $k \in \mathbb{N}$. Conversely, we will show that each such point is the landing point of uncountably many stretching rays. This result is closely related to the work of [K] stated below.

We also consider the locus $\mathcal{E}_{1}(\mathbb{R})$. As we mentioned above, the multiplier of an attracting cycle, if any, is invariant under stretching. This assures that most stretching rays land in this locus. We will show that all stretching rays in $\mathcal{E}_{1}(\mathbb{R})$ actually land by virtue of the theorem on density of hyperbolicity in the real quadratic family (cf. G-S], [Ly]).

There are very few works on the landing of stretching rays for degree greater than two. Kiwi $[\mathrm{K}]$ has considered critical portraits in the visible shift locus of complex polynomials of arbitrary degree and has characterized their impressions in terms of rational laminations. Especially, he has shown that the impression of a strictly preperiodic critical portrait consists of a single polynomial, whose critical points are strictly preperiodic. Willumsen [W] gave necessary conditions for stretching rays to accumulate on some part of $\operatorname{Per}_{1}(1)$ in the family of complex cubic polynomials. Our study is much inspired by her work. Quite recently, Buff and Henriksen [Bu-He] has also shown the existence of stretching rays (external rays) with non-trivial accumulation sets in the parameter space of the family $f_{b}(z)=$ $e^{2 \pi i \theta} z+b z^{2}+z^{3}, b \in \mathbb{C}$ where $\theta$ is a non-Brjuno number. Schleicher pointed out that he had also found that some external rays of the multicorn do not land.

Acknowledgment. The authors would like to express their hearty thanks to John Milnor and Mitsuhiro Shishikura for valuable advice. We also thank Weixiao Shen for explaining to us the notion of the radial Julia set and its relation to our problem in Subsection 6.4.

\section{Complex Cubic POlynomials}

In this paper, we study cubic polynomials as maps from the complex plane $\mathbb{C}$ to itself, and consider their iterations. In this section, we shall recall some standard definitions and notations about complex dynamics of cubic polynomials. Basic references are $[\mathrm{B}]$ and $[\mathrm{B}-\mathrm{H} 1]$.

2.1. The parameter space of complex cubic polynomials. Let $\mathcal{P}$ be the family of monic centered cubic polynomials, i.e., polynomials of the form

$$
P(z)=z^{3}-3 A z+\sqrt{B} ; \quad(A, B) \in \mathbb{C}^{2} .
$$

The polynomial $P$ is called centered if the critical points $\omega_{1}$ and $\omega_{2}$ of $P$ in $\mathbb{C}$ are central, i.e., $\omega_{1}+\omega_{2}=0$. Any cubic polynomial is complex affine conjugate to a unique polynomial of this form. We identify $\mathcal{P}$ with the $(A, B)$ parameter space

$\mathbb{C}^{2}$. Then $\mathbb{C}^{2}$ can be considered as the set of complex affine conjugacy classes of cubic polynomials.

2.2. The mappings $h_{P}$ and $\varphi_{P}$. The main tools for analyzing the dynamical behavior of a polynomial $P$ are the Green's function of the filled-in Julia set $K(P)$ and the Böttcher coordinate $\varphi_{P}$ of $P$.

We denote by $K(P)$ the set of points with bounded orbit and call it the filled-in Julia set of $P$. The boundary of $K(P)$ is called the Julia set of $P$ and is denoted by $J(P)$. We define the function $h_{P}: \mathbb{C} \rightarrow \mathbb{R}_{+} \cup\{0\}$, where $\mathbb{R}_{+}$denotes the set of 
positive real numbers, by

$$
h_{P}(z):=\lim _{n \rightarrow \infty} \frac{1}{3^{n}} \log _{+}\left|P^{n}(z)\right|,
$$

where $\log _{+}|z|:=\max \{0, \log |z|\} . h_{P}$ measures the escape rate to infinity. Then $h_{P}$ has the following properties of the Green's function of $K(P): h_{P}$ is continuous on $\mathbb{C}$, harmonic on $\mathbb{C} \backslash K(P)$, equal to 0 on $K(P)$, and $h_{P}(z)=\log |z|+O(1)$ when $|z| \rightarrow \infty$. Moreover, it satisfies the following functional equation,

$$
h_{P}(P(z))=3 \cdot h_{P}(z) \text {. }
$$

We set

$$
H(P):=\max \left\{h_{P}\left(\omega_{1}\right), h_{P}\left(\omega_{2}\right)\right\}, \quad U_{P}:=\left\{z \in \mathbb{C} \mid h_{P}(z)>H(P)\right\} .
$$

Since $\infty$ is a superattracting fixed point for any polynomial $P$, there exists a unique conformal map

$$
\varphi_{P}: U_{P} \rightarrow\left\{z \in \mathbb{C}|| z \mid>e^{H(P)}\right\}
$$

satisfying the functional equation $\varphi_{P} \circ P=P_{0} \circ \varphi_{P}$, where $P_{0}(z):=z^{3}$, with the normalization $\varphi_{P}(z) / z \rightarrow 1$ as $|z| \rightarrow \infty$. The map $\varphi_{P}$ is called the Böttcher coordinate of $P$. These two mappings are related by the identity $h_{P}(z)=\log \left|\varphi_{P}(z)\right|$ on $U_{P}$. Set $R_{P}(\theta)=\varphi_{P}^{-1}\left(\left\{z=r e^{2 \pi i \theta} \mid r>e^{H(P)}\right\}\right)$. We call it the external ray of $P$ of the external angle $\theta$ on $U_{P}$. Note that the Böttcher coordinate $\varphi_{P}$ can be analytically continued by the above functional equation. Hence each external ray can be continued until it hits a point on the backward orbits of critical points. If it never hits such a point, it accumulates on $J(P)$.

2.3. The connectedness locus and the escape locus. The parameter space $\mathcal{P}$ is decomposed into two complementary subsets; the connectedness locus $\mathcal{C}$, the set of polynomials with connected filled-in Julia set, and its complement in $\mathcal{P}$, the escape locus $\mathcal{E}$. Another characterization is that $\mathcal{C}$ consists of polynomials both of whose critical points are contained in $K(P)$, whereas $\mathcal{E}$ consists of polynomials, one of whose critical orbits is unbounded. The escape locus $\mathcal{E}$ is subdivided further:

$$
\mathcal{E}=\mathcal{E}_{1} \cup \mathcal{E}_{2}
$$

where $\mathcal{E}_{j}$ is the set of polynomials whose $j$ critical points escape to infinity.

Branner and Hubbard [B-H1] showed that the map $P \mapsto H(P)$ defines a trivial topological fibration $H: \mathcal{E} \rightarrow \mathbb{R}_{+}$where fibers $\mathcal{S}_{\rho}:=\{P \in \mathcal{E} \mid H(P)=\rho\}$ are homeomorphic to the three-dimensional spheres. Moreover, they also proved that $\mathcal{B}_{\rho}:=\{P \in \mathcal{P} \mid H(P) \leq \rho\}$ is homeomorphic to the four-dimensional closed ball. Then the identity $\mathcal{C}=\bigcap_{\rho>0} \mathcal{B}_{\rho}$ implies that $\mathcal{C}$ is compact and cellular, hence connected and full.

2.4. The wring construction and stretching rays. Let $u=s+i t \in \mathbb{H}_{+}$be any complex number in the right half plane, i.e., $s>0$. We shall associate to $u$ a polynomial $P_{u}$ obtained from $P$ by wringing the complex structure as follows.

The map $f_{u}(z):=z|z|^{u-1}$ is a quasiconformal diffeomorphism from $\{z \in \mathbb{C} \mid$ $|z|>1\}$ to itself commuting with $P_{0}(z)=z^{3}$. Recall that the Böttcher coordinate $\varphi_{P}$ conjugates $P$ to $P_{0}$ on $U_{P}$. Let $\sigma_{u}$ denote the $P$-invariant almost complex structure on $\mathbb{C}$ satisfying

$$
\sigma_{u}:=\left\{\begin{array}{l}
\left(f_{u} \circ \varphi_{P}\right)^{*} \sigma_{0} \\
\sigma_{0} \text { on } K(P)
\end{array} \text { on } U_{P}\right.
$$


where $\sigma_{0}$ denotes the standard complex structure on $\mathbb{C}$. By the Measurable Riemann Mapping Theorem, there exists a unique quasiconformal map $F_{u}$ on $\mathbb{C}$ satisfying

$$
F_{u}^{*} \sigma_{0}=\sigma_{u}, \quad \lim _{z \rightarrow \infty} \frac{f_{u} \circ \varphi_{P} \circ F_{u}^{-1}(z)}{z}=1, \quad P_{u}:=F_{u} \circ P \circ F_{u}^{-1} \in \mathcal{P} .
$$

Since $F_{u}$ depends holomorphically on $u$, so does $P_{u}$. Thus we define a holomorphic map $W_{P}: \mathbb{H}_{+} \rightarrow \mathcal{P}$ by $W_{P}(u)=P_{u}$. Then the Böttcher coordinate $\varphi_{P_{u}}$ of $P_{u}$ is equal to $f_{u} \circ \varphi_{P} \circ F_{u}^{-1}$. This operation is called the wringing.

We should remark that $P_{u}$ is hybrid equivalent to $P$ from the definition of $\sigma_{u}$. Hence it holds $P_{u}=P$ for $P \in \mathcal{C}$ by the straightening theorem in $[\mathrm{D}-\mathrm{H}]$.

For $P \in \mathcal{E}$, we define the stretching ray through $P$ by

$$
R(P):=W_{P}\left(\mathbb{R}_{+}\right)=\left\{P_{s} \mid s>0\right\} .
$$

Since $\left|\varphi_{P_{s}}(z)\right|=\left|f_{s} \circ \varphi_{P} \circ F_{s}^{-1}(z)\right|=\left|\varphi_{P} \circ F_{s}^{-1}(z)\right|^{s}$, we have $h_{P_{s}}(z)=s \cdot h_{P}\left(F_{s}^{-1}(z)\right)$ and $H\left(P_{s}\right)=s \cdot H(P)$ for $s>0$. Thus $R(P)$ actually forms a ray such that $P_{s}$ escapes to infinity if $s \rightarrow+\infty$ while it accumulates on $\partial \mathcal{C}$, the boundary of the connectedness locus, if $s \rightarrow 0$. Moreover, $R(P) \cap R(Q) \neq \emptyset$ implies $R(P)=R(Q)$.

We should remark again that any map in $R(P)$ is hybrid equivalent to $P$, which is crucial in our arguments below.

2.5. Invariants on stretching rays. To analyze accumulation sets of stretching rays, it is helpful to find well-behaved invariants on stretching rays. For $P \in \mathcal{E}_{2}$, we define the Böttcher vector $\eta(P)$ by $\eta(P):=\frac{1}{\log 3} \log \frac{h_{P}\left(\omega_{2}\right)}{h_{P}\left(\omega_{1}\right)}$, where $\omega_{j}=\omega_{j}(P)$, $j=1,2$ are two escaping critical points of $P$. Then, it easily follows that

$$
\eta\left(P_{s}\right)=\frac{1}{\log 3} \log \frac{h_{P_{s}}\left(F_{s}\left(\omega_{2}\right)\right)}{h_{P_{s}}\left(F_{s}\left(\omega_{1}\right)\right)}=\frac{1}{\log 3} \log \frac{h_{P}\left(\omega_{2}\right)}{h_{P}\left(\omega_{1}\right)}=\eta(P) .
$$

(Later we set $\omega_{1}=\sqrt{A}, \omega_{2}=-\sqrt{A}$.) Thus we get the following.

Proposition 2.1. For $P \in \mathcal{E}_{2}$, the Böttcher vector $\eta\left(P_{s}\right)$ of $P_{s} \in R(P)$ is invariant on $R(P)$.

For $P \in \mathcal{E}_{1}$ we must consider other invariants on stretching rays and it is not easy to find them. On the other hand, if $P$ has an attracting or a parabolic cycle of period $k$ with multiplier $\lambda$, then $k$ and $\lambda$ are invariant on $R(P)$ since any map in $R(P)$ is hybrid equivalent to $P$.

Proposition 2.2. For $P \in \mathcal{E}_{1}$, if $P$ has an attracting or a parabolic cycle of period $k$ with multiplier $\lambda$, then $k$ and $\lambda$ are invariant on $R(P)$.

\section{ReAl CUBiC POLYNOMials}

In this section, we shall restrict our attention to real cubic polynomials whose critical points are both real. Basic reference for the parameter space of real cubic polynomials is Mi1. 


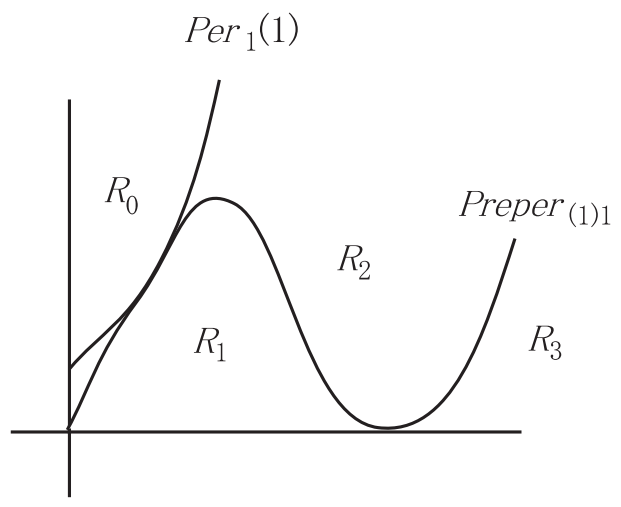

Figure 3. Partition of the parameter space

3.1. The parameter space of real cubic polynomials. The parameter space of monic centered cubic polynomials with real coefficients is the closed upper half plane of the real $(A, B)$-plane $\mathbb{R}^{2}$. Moreover, we assume that our real cubic map is bimodal; in other words, its critical points are both real and distinct. We do not consider the positive $A$-axis, since $\{B=0, A>1\}$ forms a stretching ray which trivially lands at $(1,0)$ while $\{B=0, A \in[0,1]\}$ is contained in the connectedness locus. Then the set of these maps corresponds to $\mathbb{R}_{+}^{2}$, the first quadrant of $\mathbb{R}^{2}$.

Because a real polynomial can be also considered as a map from the real axis $\mathbb{R}$ to itself, Milnor introduced a partition of the parameter space $\mathbb{R}_{+}^{2}$ into four regions, $\mathcal{R}_{0}, \mathcal{R}_{1}, \mathcal{R}_{2}, \mathcal{R}_{3}: P$ belongs to $\mathcal{R}_{0}$ if $K(P) \cap \mathbb{R}$, the real locus of the filled-in Julia set of $P$, consists of one fixed point of $P$. Let $I$ be the smallest closed interval containing $K(P) \cap \mathbb{R}$. Then for $P \notin \mathcal{R}_{0}, P$ belongs to $\mathcal{R}_{j}$ if the graph of $P$ intersected with $I \times I$ has $j$ distinct components. The following easily follows from the definition.

Proposition 3.1. $\mathcal{R}_{1}$ is equal to $\mathcal{C}(\mathbb{R}):=\mathcal{C} \cap \mathbb{R}_{+}^{2} \cdot \mathcal{R}_{0}$ and $\mathcal{R}_{3}$ are contained in $\mathcal{E}_{2}(\mathbb{R}):=\mathcal{E}_{2} \cap \mathbb{R}_{+}^{2}$, whereas $\mathcal{R}_{2}$ consists of both elements of $\mathcal{E}_{1}(\mathbb{R}):=\mathcal{E}_{1} \cap \mathbb{R}_{+}^{2}$ and $\mathcal{E}_{2}(\mathbb{R})$.

The following real algebraic curves play special roles in the parameter space $\mathbb{R}_{+}^{2}$ :

$$
\begin{aligned}
\operatorname{Per}_{1}(1) & : B=4(A+1 / 3)^{3}, \\
\operatorname{Preper}_{(1) 1}: B & =4 A(A-1)^{2} .
\end{aligned}
$$

The boundary $\partial \mathcal{C}(\mathbb{R})$ of $\mathcal{C}(\mathbb{R})$ consists of $\operatorname{Per}_{1}(1)$ where $0 \leq A \leq 1 / 9$ and $\operatorname{Preper}_{(1) 1}$ where $1 / 9 \leq A \leq 1$.

The locus $\operatorname{Per}_{1}(1)$ is the set of parameters which has a parabolic fixed point with multiplier one, while the locus $\operatorname{Preper}_{(1) 1}$ is the set of parameters, one of whose critical values is a fixed point. $\operatorname{Per}_{1}(1)$ is the boundary between $\mathcal{R}_{0}$ and $\mathcal{R}_{1} \cup \mathcal{R}_{2}$, whereas $\operatorname{Preper}_{(1) 1}$ contains the boundary between $\mathcal{R}_{2}$ and $\mathcal{R}_{1} \cup \mathcal{R}_{3}$; see Figure 3

In the region $B<4(A+1 / 3)^{3}$ corresponding to $\mathcal{R}_{1} \cup \mathcal{R}_{2} \cup \mathcal{R}_{3}, P$ has three distinct real fixed points, which we denote by $\beta_{P}^{\prime}<\beta_{P}^{\prime \prime}<\beta_{P}$. Then it is easy to see $I=\left[\beta_{P}^{\prime}, \beta_{P}\right]$. On $\operatorname{Per}_{1}(1): B=4(A+1 / 3)^{3}, \beta_{P}=\beta_{P}^{\prime \prime}=\sqrt{A+1 / 3}$ is the parabolic fixed point with multiplier one. In the region $B>4(A+1 / 3)^{3}$ corresponding to $\mathcal{R}_{0}, P$ has only one real fixed point $\beta_{P}^{\prime}$; see Figure 4 . 


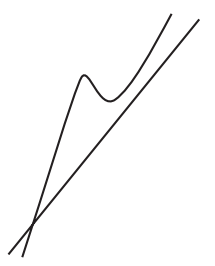

$\beta P^{\prime}$
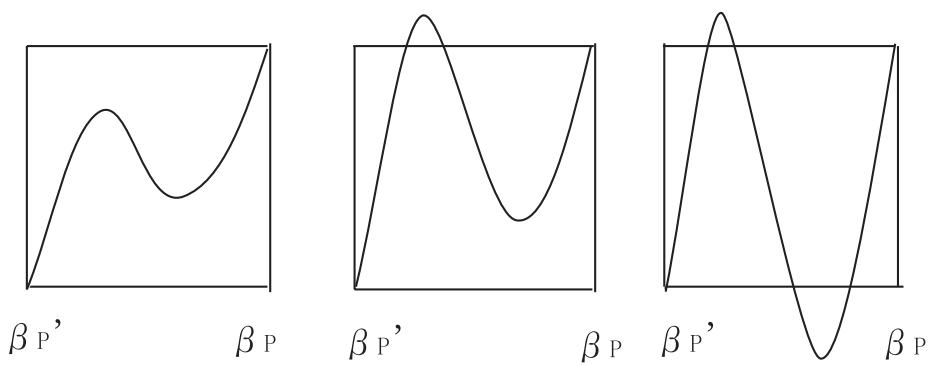

FIGURE 4. Graphs of cubic maps: from left to right, $\mathcal{R}_{0}, \mathcal{R}_{1}, \mathcal{R}_{2}, \mathcal{R}_{3}$

Lemma 3.2. The locus Preper $_{(1) 1}: B=4 A(A-1)^{2}$ is expressed by $P(-\sqrt{A})=\beta_{P}^{\prime \prime}$ if $A \leq 1 / 9, P(-\sqrt{A})=\beta_{P}$ if $1 / 9 \leq A \leq 1$ and $P(\sqrt{A})=\beta_{P}^{\prime}$ if $A \geq 1$.

Proof. Put $A=a^{2}$, i.e., $a=\sqrt{A}$ or $-\sqrt{A}$ and $b=\sqrt{B}$. Since the critical value $P(a)$ of $P(z)=z^{3}-3 a^{2} z+b$ satisfies $P(a)=P(-2 a)=b-2 a^{3}, P(a)$ must be equal to $-2 a$ if $P(a)$ is a fixed point of $P$ while $a$ is not a fixed point of $P$. Thus $\operatorname{Preper}_{(1) 1}$ is expressed by $b=2 a\left(a^{2}-1\right)$. Since $b=\sqrt{B}>0$ in our parameter space, we have $a>1$ or $-1<a<0$. Hence, if $0<A<1, a=-\sqrt{A}$ and $P(-\sqrt{A})=\beta_{P}$ or $\beta_{P}^{\prime \prime}$. Note that the multiplier of $-2 a$ is $9 a^{2}$. Hence, if $A<1 / 9$, $-2 a$ is attracting and must be equal to $\beta_{P}^{\prime \prime}$, that is, $P(-\sqrt{A})=\beta_{P}^{\prime \prime}$. If $1 / 9 \leq A<1$, $-2 a$ is repelling and must be $\beta_{P}$, that is, $P(-\sqrt{A})=\beta_{P}$. If $A \geq 1, a=\sqrt{A}$ and $P(\sqrt{A})=\beta_{P}^{\prime}$. This completes the proof.

Now let us briefly look at the complex dynamical plane. For $P \in \mathcal{R}_{0}, J(P)$ is a Cantor set and intersects with $\mathbb{R}$ only at $\beta_{P}^{\prime}$. Both rays $R_{P}(0)$ and $R_{P}(1 / 2)$ land at $\beta_{P}^{\prime}$. For $P \in \mathcal{R}_{3}, J(P)$ is a Cantor set contained in $\mathbb{R}$. In $\mathcal{R}_{1}, J(P)$ is connected, while $J(P)$ is disconnected but not necessarily a Cantor set in $\mathcal{R}_{2}$.

\subsection{Critical orbits of $P \in \mathbb{R}_{+}^{2} \backslash \mathcal{C}(\mathbb{R})$.}

Lemma 3.3. For $P \in \mathbb{R}_{+}^{2} \backslash \mathcal{C}(\mathbb{R}), H(P)=h_{P}(-\sqrt{A})$. That is, the critical point $-\sqrt{A}$ escapes faster than another critical point $\sqrt{A}$.

Proof. Since $P(-\sqrt{A})>P(\sqrt{A})>\sqrt{A}$ in $\mathcal{R}_{0}$, we have $P^{k}(-\sqrt{A})>P^{k}(\sqrt{A})$ for any $k$ and the conclusion follows. In the region $\mathcal{R}_{2}, P(-\sqrt{A})>\beta_{P}>P(\sqrt{A})$ holds; hence it follows that $P^{k}(-\sqrt{A})>P^{k}(\sqrt{A})$ also for any $k \geq 1$.

We will show $\left|P^{k}(-\sqrt{A})\right|>\left|P^{k}(\sqrt{A})\right|$ in the region $\mathcal{R}_{3}$. Put $x_{k}=P^{k}(-\sqrt{A})$; $y_{k}=P^{k}(\sqrt{A})$. Since $\sqrt{B}<2 \sqrt{A}(A-1)$ in $\mathcal{R}_{3}, y_{1}<-2 \sqrt{A}$ and $x_{1}>2 A \sqrt{A}$. Moreover, $x_{k}$ is strictly increasing, while $y_{k}$ is strictly decreasing. By induction on $k$, we show that $x_{k}>-y_{k}$. Obviously $x_{1}>-y_{1}$. Suppose $x_{k}>-y_{k}$. Since the function $x \mapsto x^{3}-3 A x$ is increasing in $x>\sqrt{A}$, it follows that

$$
\begin{aligned}
x_{k+1} & =P\left(x_{k}\right)=x_{k}^{3}-3 A x_{k}+\sqrt{B}>-y_{k}^{3}+3 A y_{k}+\sqrt{B} \\
& =-P\left(y_{k}\right)+2 \sqrt{B}=-y_{k+1}+2 \sqrt{B}>-y_{k+1} .
\end{aligned}
$$

This completes the proof.

The next result easily follows from Figure 4. 
Lemma 3.4. For $P \in \mathcal{R}_{2}$, the orbit of a point $x>\beta_{P}^{\prime}(x=\sqrt{A}$ in particular $)$ escapes to $\infty$ if and only if there exists $k \geq 0$ such that $P^{k}(x)>\beta_{P}$. In this case, $P^{n}(x) \rightarrow+\infty$.

It is easy to see that in $\mathcal{R}_{3}, P(\sqrt{A})<\beta_{P}^{\prime}$ and $P^{n}(\sqrt{A}) \rightarrow-\infty$. In $\mathcal{R}_{0}$, $P^{n}(\sqrt{A}) \rightarrow+\infty$.

From Lemmas 3.4 and 3.3, it follows that

$$
\mathcal{E}_{1}(\mathbb{R})=\left\{(A, B) \in \mathcal{R}_{2} \mid P^{k}(\sqrt{A}) \leq \beta_{P} \text { for any } k \geq 0\right\} .
$$

For any connected component $U$ of $\mathcal{E}_{2}(\mathbb{R}) \cap \mathcal{R}_{2}$, there exists a unique $k \in \mathbb{N}$ such that

$$
P^{k}(\sqrt{A})<\beta_{P}<P^{k+1}(\sqrt{A}),
$$

in $U$, since $P$ is monotone increasing in $x>\beta_{P}$. Hence its boundary $\partial U$ is contained in the real analytic set $P^{k+1}(\sqrt{A})=\beta_{P}$.

\subsection{Stretching rays in $\mathbb{R}_{+}^{2}$.}

Proposition 3.5. For $P \in \mathbb{R}_{+}^{2}$, the stretching ray $R(P)$ is contained in $\mathbb{R}_{+}^{2}$.

Proof. $\varphi_{P}$ and $f_{s}$ commute with complex conjugation, hence so does $F_{s}$, and $P_{s} \in$ $\mathbb{R}_{+}^{2}$.

The following is a trivial case.

Lemma 3.6. $\operatorname{Per}_{1}(1) \cap\left(\mathbb{R}_{+}^{2} \backslash \mathcal{C}(\mathbb{R})\right)$ and Preper $_{(1) 1} \cap\left(\mathbb{R}_{+}^{2} \backslash \mathcal{C}(\mathbb{R})\right)$ are stretching rays, landing at $\left(1 / 9,4^{4} / 9^{3}\right)$ and at $(1,0)$ respectively.

Since two distinct stretching rays are disjoint, we roughly understand the location of accumulation sets of stretching rays from Lemma 3.6 and Figure 3 .

Proposition 3.7. Let $P$ belong to $\mathbb{R}_{+}^{2} \backslash \mathcal{C}(\mathbb{R})$.

(1) For $P \in \mathcal{R}_{0}, R(P)$ accumulates on the Per $1(1)$-part of $\partial \mathcal{C}(\mathbb{R})$.

(2) For $P \in \mathcal{R}_{2}, R(P)$ accumulates on the $\operatorname{Preper}_{(1) 1}$-part of $\partial \mathcal{C}(\mathbb{R})$.

(3) For $P \in \mathcal{R}_{3}, R(P)$ lands at $(A, B)=(1,0)$.

Consequently, nothing remains to be done in $\mathcal{R}_{3}$, hence in the following sections we just consider the landing problem of stretching rays in $\mathcal{R}_{0}$ and $\mathcal{R}_{2}$.

3.4. Böttcher vectors in the real shift locus. We set $\zeta_{P}(z):=\log \log \varphi_{P}(z) /$ $\log 3$. We can characterize the Böttcher vector in terms of $\zeta_{P}$, which will play an essential role in the sequel.

Lemma 3.8. For any $P \in \mathcal{E}_{2}(\mathbb{R})$, there exists $k \in \mathbb{N}$ such that for any $n \geq k$,

$$
\eta(P)=\zeta_{P}\left(P^{n}(-\sqrt{A})\right)-\zeta_{P}\left(P^{n}(\sqrt{A})\right) .
$$

In particular, $\eta(P)$ is a positive real number.

Proof. By Lemma 3.4, for any $P \in \mathcal{E}_{2}(\mathbb{R}) \cap \mathcal{R}_{2}$, there exists $k$ such that $P^{k}(\sqrt{A})>$ $\beta_{P}$. Since the external ray of angle 0 for $P$ is the ray $\left\{x>\beta_{P}\right\}$ on the real axis, it follows that $\varphi_{P}\left(P^{n}( \pm \sqrt{A})\right)>1$ is well defined for any $n \geq k$. For $P \in \mathcal{R}_{0}$, the ray $\{x>\sqrt{A}\}$ on the real axis forms a part of the external ray $R_{P}(0)$ of angle 0 . Thus $\varphi_{P}(P( \pm \sqrt{A}))>1$ is well defined and we can take $k=1$. Then $\zeta_{P}\left(P^{n}( \pm \sqrt{A})\right)$ is well defined and satisfies

$$
\zeta_{P}\left(P^{n}( \pm \sqrt{A})\right)=\log h_{P}\left(P^{n}( \pm \sqrt{A})\right) / \log 3=\log h_{P}( \pm \sqrt{A}) / \log 3+n
$$

for $P \in \mathcal{R}_{0} \cup\left(\mathcal{E}_{2}(\mathbb{R}) \cap \mathcal{R}_{2}\right)$. Hence the above equality follows. 
Remark 3.9. Willumsen [W] has shown that, if $R(P)$ accumulates a point $Q \in$ $\operatorname{Per}_{1}(1), R_{P}(0)$ bifurcates at a critical point and conjectured that another critical point also hits $R_{P}(0)$ after a finite iteration. These two conditions are always satisfied for $P \in \mathcal{R}_{0}$ (cf. Proposition 3.7).

\section{LANDING PROPERTY OF STRETCHING RAYS IN $\mathcal{E}_{2}(\mathbb{R}) \cap \mathcal{R}_{2}$}

In this section, we consider the landing of stretching rays in the real shift locus $\mathcal{E}_{2}(\mathbb{R})$ in the region $\mathcal{R}_{2}$. Since both critical points are real, we mainly consider the dynamics only on the real axis.

Let $U$ be a connected component of $\mathcal{E}_{2}(\mathbb{R}) \cap \mathcal{R}_{2}$. As we remarked in Subsection 3.2 there is a unique $k \in \mathbb{N}$ satisfying

$$
P^{k}(\sqrt{A})<\beta_{P}<P^{k+1}(\sqrt{A}),
$$

for any $P \in U$ and its boundary $\partial U$ is contained in the real analytic set $P^{k+1}(\sqrt{A})$ $=\beta_{P}$.

Lemma 4.1. Every stretching ray in such a component $U$ lands at a point $P_{0}=$ $\left(A_{0}, B_{0}\right) \in \operatorname{Preper}_{(1) 1} \cap \partial \mathcal{C}(\mathbb{R})$ satisfying $P_{0}^{k}\left(\sqrt{A_{0}}\right)=-\sqrt{A_{0}}$.

Proof. For $P \in U, P^{k}(\sqrt{A})$ satisfies $\alpha_{P}<P^{k}(\sqrt{A})<\alpha_{P}^{\prime}$, where $\alpha_{P}$ and $\alpha_{P}^{\prime}$ are preimages of $\beta_{P}$ other than $\beta_{P}$. This relation is preserved under stretching. As $P$ approaches $\operatorname{Preper}_{(1) 1} \cap \partial \mathcal{C}(\mathbb{R})$, both points $\alpha_{P}$ and $\alpha_{P}^{\prime}$ tends to $-\sqrt{A}$. Hence any accumulation point of the stretching rays in $U$ satisfies $P^{k}(\sqrt{A})=-\sqrt{A}$. It defines a real analytic curve, which intersects the semi-algebraic curve $\operatorname{Preper}_{(1) 1} \cap \partial \mathcal{C}(\mathbb{R})$ only at finite points. Thus the stretching ray in $U$ must land at a point $P_{0}=$ $\left(A_{0}, B_{0}\right) \in \operatorname{Preper}_{(1) 1}$ satisfying $P_{0}^{k}\left(\sqrt{A_{0}}\right)=-\sqrt{A_{0}}$.

Next we will show the converse statement. To show this, we need a lemma.

Lemma 4.2. Suppose $P_{0}=\left(A_{0}, B_{0}\right) \in \mathbb{R}_{+}^{2}$ satisfies $P_{0}^{k}\left(\sqrt{A_{0}}\right)=-\sqrt{A_{0}}$. Then $(\partial / \partial B)\left(P^{k+1}(\sqrt{A})-\beta_{P}\right)>0$ at $\left(A_{0}, B_{0}\right)$.

Proof. Differentiating both sides of $P\left(\beta_{P}\right)=\beta_{P}$ with respect to $B$, it follows that

$$
\frac{\partial \beta_{P}}{\partial B}=-\frac{1}{2 \sqrt{B}\left(P^{\prime}\left(\beta_{P}\right)-1\right)}<0
$$

Hence, we have only to show $(\partial / \partial B)\left(P^{k+1}(\sqrt{A})\right)>0$ at $\left(A_{0}, B_{0}\right)$. From the assumption, it follows at $\left(A_{0}, B_{0}\right)$ :

$$
\begin{aligned}
\left.(\partial / \partial B) P^{k+1}(\sqrt{A})\right|_{\left(A_{0}, B_{0}\right)} & =\left.(\partial / \partial B) P\left(P^{k}(\sqrt{A})\right)\right|_{\left(A_{0}, B_{0}\right)} \\
& =\frac{1}{2 \sqrt{B_{0}}}+\left.P_{0}^{\prime}\left(P_{0}^{k}\left(\sqrt{A_{0}}\right)\right) \cdot(\partial / \partial B) P^{k}(\sqrt{A})\right|_{\left(A_{0}, B_{0}\right)} \\
& =\frac{1}{2 \sqrt{B_{0}}}>0 .
\end{aligned}
$$

This completes the proof.

Proposition 4.3. The point $P_{0}=\left(A_{0}, B_{0}\right) \in \operatorname{Preper}_{(1) 1}$ satisfying $P_{0}^{k}\left(\sqrt{A_{0}}\right)=$ $-\sqrt{A_{0}}$ is a landing point of two stretching rays of the form $P^{k+1}(\sqrt{A})=\beta_{P}$. They are tangent to $\operatorname{Preper}_{(1) 1} \cap \partial \mathcal{C}(\mathbb{R})$ at $P_{0}$. This equation defines a real analytic curve through $P_{0}$, which forms the boundary of a connected component of $\mathcal{E}_{2}(\mathbb{R})$ consisting of $P$ satisfying (4.1). 


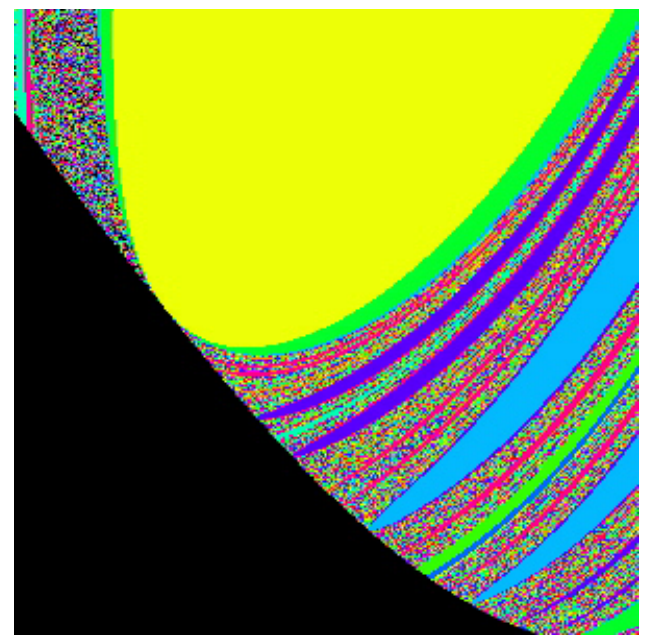

FIgURE 5. Stretching rays tangent to $\partial \mathcal{C}(\mathbb{R})$

Proof. By Lemma 4.2 and the implicit function theorem, it follows that there exists locally a unique real analytic curve expressed by $P^{k+1}(\sqrt{A})-\beta_{P}=0$ passing through $P_{0}$. Since $P^{k+1}(\sqrt{A})>\beta_{P}$ holds above this curve, this curve forms the boundary of a connected component $U$ of $\mathcal{E}_{2}(\mathbb{R})$. Since it is not contained in $\partial \mathcal{C}(\mathbb{R})$, it gives two stretching rays landing at $P_{0}$. Since $\operatorname{Preper}_{(1) 1} \cap \partial \mathcal{C}(\mathbb{R})$ is also smooth at $P_{0}$, these two rays are tangent to $\partial \mathcal{C}(\mathbb{R})$ at $P_{0}$.

Suppose that $P^{k}(\sqrt{A}) \geq \beta_{P}$ for some $P \in U$. Then the landing point $P_{0}$ of $R(P)$ must satisfy $P_{0}^{k}\left(\sqrt{A_{0}}\right)=\beta_{P_{0}}$, which contradicts the assumption that $P_{0}^{k}\left(\sqrt{A_{0}}\right)=$ $-\sqrt{A_{0}}$. Thus $P^{k}(\sqrt{A})<\beta_{P}$ holds for $P \in U$.

Summarizing results in this section, we have the following.

Theorem 4.4. For any $k \in \mathbb{N}$, there is a one-to-one correspondence between connected components $U$ of $\mathcal{E}_{2}(\mathbb{R}) \cap \mathcal{R}_{2}$ of the form:

$$
P^{k}(\sqrt{A})<\beta_{P}<P^{k+1}(\sqrt{A})
$$

and points $P_{0}=\left(A_{0}, B_{0}\right)$ on Preper $_{(1) 1} \cap \partial \mathcal{C}(\mathbb{R})$ satisfying $P_{0}^{k}\left(\sqrt{A_{0}}\right)=-\sqrt{A_{0}}$ such that the stretching ray $R(P)$ for any $P \in U$ lands at $P_{0}$. The boundary of $U$ consists of two stretching rays satisfying $P^{k+1}(\sqrt{A})=\beta_{P}$, which land tangentially at $P_{0}$.

Figure 5 is an enlargement of Figure 1. This supports the above result.

Remark 4.5. Theorem 4.4 gives examples where uncountably many stretching rays land at the same point.

Remark 4.6. In general, there are many connected components of $\mathcal{E}_{2}(\mathbb{R}) \cap \mathcal{R}_{2}$ satisfying (4.1). For example, for $k=2,3$, there are exactly two components.

Note that the region $\mathcal{R}_{2}$ seems to be disjoint from the visible shift locus, hence our result will not be included in that of $[\mathrm{K}]$. Roughly speaking, a map in the shift locus is visible if each critical point is the terminating point of some external rays and its external angles are well defined. As for a precise definition, see $[\mathrm{K}]$. 


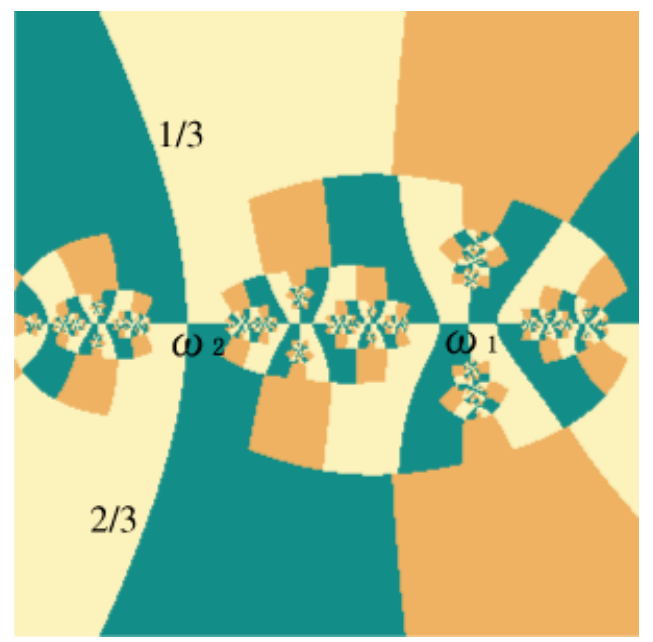

FiguRE 6. Julia set of a map in $\mathcal{E}_{2}(\mathbb{R}) \cap \mathcal{R}_{2}$

Figure 6 is the Julia set of a map in $\mathcal{E}_{2}(\mathbb{R}) \cap \mathcal{R}_{2}$. It presents a ternary decomposition of the complement of the filled-in Julia set. We can see some external rays and critical points. It suggests that external rays of angles $1 / 3$ and $2 / 3$ terminate at the critical point $\omega_{2}=-\sqrt{A}$, while no external rays terminate at another critical point $\omega_{1}=\sqrt{A}$.

\section{LANDing PROPERTY OF STRETCHING RAYS IN $\mathcal{E}_{1}(\mathbb{R})$}

In this section, we investigate the landing of stretching rays in $\mathcal{E}_{1}(\mathbb{R})$. From Subsection 3.3, $R(P)$ for $P \in \mathcal{E}_{1}(\mathbb{R})$ accumulates on $\operatorname{Preper}_{(1) 1} \cap \partial \mathcal{C}(\mathbb{R})$. Recall that only one critical point escapes for $P=(A, B) \in \mathcal{E}_{1}(\mathbb{R})$, which is equal to $-\sqrt{A}$ by Lemma 3.3 Hence $\sqrt{A}$ is contained in $K(P)$.

5.1. Hyperbolic rays land. Let $\operatorname{Per}_{k}(\lambda)$ denote the set of maps $P$ having an attracting $k$-cycle with multiplier $\lambda$. Assume that $P \in \mathcal{E}_{1}(\mathbb{R})$ belongs to $\operatorname{Per}_{k}(\lambda)$. Then $k$ and $\lambda$ is constant on $R(P)$ by Proposition 2.2. Hence $R(P) \subset \operatorname{Per}_{k}(\lambda)$. Let $P_{0} \in \operatorname{Preper}_{(1) 1} \cap \partial \mathcal{C}(\mathbb{R})$ be an accumulation point of $R(P)$. Then $P_{0}$ is also contained in $\operatorname{Per}_{k}(\lambda)$. Since $\operatorname{Per}_{k}(\lambda)$ is a real analytic set, its intersection with the semi-algebraic set $\operatorname{Preper}_{(1) 1} \cap \partial \mathcal{C}(\mathbb{R})$ is locally finite. Thus $R(P)$ must land at a point $P_{0} \in \operatorname{Preper}_{(1) 1}$. Hence we proved the following result.

Proposition 5.1. If $P \in \mathcal{E}_{1}(\mathbb{R})$ belongs to $\operatorname{Per}_{k}(\lambda)$, then $R(P)$ lands at a point $P_{0} \in \operatorname{Preper}_{(1) 1} \cap \partial \mathcal{C}(\mathbb{R})$, which is also contained in $\operatorname{Per}_{k}(\lambda)$.

5.2. Hyperbolic parameters are landing points. To prove the converse, we need the following lemma. Here we call a map on Preper $_{(1) 1}$ hyperbolic if it has an attracting cycle.

Lemma 5.2. The multiplier map is real valued and monotone on each hyperbolic component of Preper ${ }_{(1) 1}$.

Proof. Since every attracting cycle attracts a critical orbit, which lies on the real axis, the attracting cycle must be real. Hence its multiplier is also real. To show the 


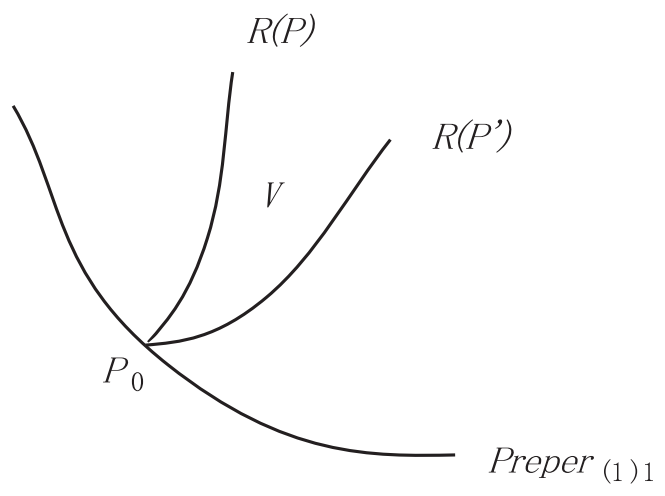

FiguRe 7. Two rays land at one point

monotonicity, we complexify the parameter space $\operatorname{Preper}_{(1) 1}$. Then, the multiplier map is continued to the complexified hyperbolic component $U$. By the standard qc-surgery method (cf. [C-G, Theorem 2.1 in Chapter 8]), we can show that the multiplier map is a conformal isomorphism between $U$ and the open unit disk. Restricting it to the real axis, we get the monotonicity.

Proposition 5.3. If $P_{0} \in \operatorname{Preper}_{(1) 1} \cap \partial \mathcal{C}(\mathbb{R})$ belongs to $\operatorname{Per}_{k}(\lambda)$, it is the landing point of a unique stretching ray $R(P)$, where $P \in \operatorname{Per}_{k}(\lambda)$.

Proof. First we show the existence of stretching rays landing at $P_{0}$. By the implicit function theorem, any map in a neighborhood of $P_{0}$ also has an attracting $k$-cycle. By Lemma 5.2, there exist $P_{ \pm} \in \operatorname{Preper}_{(1) 1} \cap \partial \mathcal{C}(\mathbb{R})$ with multiplier $\lambda \pm \epsilon$ around $P_{0}$. Here $\epsilon$ is a small positive constant. On any arc joining $P_{+}$and $P_{-}$in the complement of $\mathcal{C}(\mathbb{R})$, there must be a map $P \in \operatorname{Per}_{k}(\lambda)$. Thus there is a sequence in $\mathcal{E}_{1}(\mathbb{R}) \cap \operatorname{Per}_{k}(\lambda)$ tending to $P_{0}$. Since $\operatorname{Per}_{k}(\lambda)$ is a real analytic set, it has a connected component through $P_{0}$. This component yields a stretching ray landing at $P_{0}$.

Next we show the uniqueness of the stretching rays landing at $P_{0}$. Suppose there exists another stretching ray $R\left(P^{\prime}\right)$ also landing at $P_{0}$. Let $V$ be the region bounded by $R(P)$ and $R\left(P^{\prime}\right)$; see Figure 7. Stretching rays in $V$ must land at $P_{0}$. Then, by Proposition 5.1, the intersection of $V$ and a small open neighborhood of $P_{0}$ is contained in $\operatorname{Per}_{k}(\lambda)$. That is, the multiplier map is constant there. Hence it is constant everywhere, since it is real analytic. This is a contradiction. This completes the proof.

5.3. Non-hyperbolic rays also land. Assume that $P \in \mathcal{E}_{1}(\mathbb{R})$ has no attracting cycles. We will show that $R(P)$ also lands at a map on $\operatorname{Preper}_{(1) 1}$. For our proof, the theorem on density of hyperbolicity in the real quadratic family is crucial.

Proposition 5.4. The stretching ray $R(P)$ through any $P \in \mathcal{E}_{1}(\mathbb{R})$ lands at some point on Preper $_{(1) 1}$.

Proof. We may assume that $P$ has no attracting cycles. Branner and Douady [B-D] have shown that there exists a homeomorphism between $\mathcal{M}_{1 / 2}$, the $1 / 2$-limb of the Mandelbrot set $\mathcal{M}$ and the limb $F_{+}$of the connectedness locus $F$ of the complex 
parameter space $\operatorname{Preper}_{(1) 1}$. Restricted to the real axis, this gives a homeomorphism between $\mathcal{M}_{1 / 2} \cap \mathbb{R}$ and $F_{+} \cap \mathbb{R}$, the $\operatorname{Preper}_{(1) 1}$-part of $\partial \mathcal{C}(\mathbb{R})$. On the other hand, Graczyk-Świątek [G-S] and Lyubich $[\mathrm{Ly}$ have shown that hyperbolic maps are dense in $\mathcal{M} \cap \mathbb{R}$. Hence by means of this isomorphism between the real locus of $\mathcal{M}_{1 / 2}$ and $\operatorname{Preper}_{(1) 1} \cap \partial \mathcal{C}(\mathbb{R})$, we can show that the set of maps having attracting cycles are also dense in our family $\operatorname{Preper}_{(1) 1} \cap \partial \mathcal{C}(\mathbb{R})$. Now, since the accumulation set $I(P)=\overline{R(P)}-R(P)$ of $R(P)$ is a compact connected set of $\operatorname{Preper}_{(1) 1}$, it must be a closed subarc. If it is not a single point, it includes a hyperbolic subarc. By Proposition 5.3, each point on this subarc is the landing point of a unique stretching ray. This is a contradiction. Thus $I(P)$ must be a single point and $R(P)$ lands at that point.

5.4. Non-hyperbolic parameters are also landing points. To show the converse, we will use the following notations from $[\overline{\mathrm{B}}]$. For a complex cubic map $P \in \mathcal{E}$, we denote its critical points by $\omega_{1}$ and $\omega_{2}$. We may assume $h_{P}\left(\omega_{2}\right) \geq h_{P}\left(\omega_{1}\right)$; that is, $H(P)=h_{P}\left(\omega_{2}\right)$. We set

$$
\begin{aligned}
\mathcal{S}_{\rho} & :=\{P \in \mathcal{E} \mid H(P)=\rho\}, \\
\mathcal{H}_{\rho} & :=\left\{P \in \mathcal{S}_{\rho} \mid h_{P}\left(\omega_{1}\right)<\rho\right\} .
\end{aligned}
$$

Let $\omega_{2}^{\prime}$ be the co-critical point of $\omega_{2}$, i.e., it satisfies $P\left(\omega_{2}^{\prime}\right)=P\left(\omega_{2}\right)$. For $P \in \mathcal{S}_{\rho}$ and $\theta \in \mathbb{R} / \mathbb{Z}$, put $\Phi_{\rho}(P):=\frac{\varphi_{P}\left(\omega_{2}^{\prime}\right)}{\left|\varphi_{P}\left(\omega_{2}^{\prime}\right)\right|}$ and

$$
F_{\rho}(\theta):=\left\{P \in \mathcal{H}_{\rho} \mid \Phi_{\rho}(P)=e^{2 \pi i \theta}\right\} .
$$

Theorem 9.1 in [B-H2] says that, in the complex parameter space, $\mathcal{E}_{1} \cap F_{\rho}(\theta)$ is either a single point or a homeomorphic copy of the Mandelbrot set.

In our case, $\omega_{2}=-\sqrt{A}, \omega_{1}=\sqrt{A}$ and $\omega_{2}^{\prime}=2 \sqrt{A}>\beta_{P}$. Hence, it is easy to see that $\Phi_{\rho}(P)=1$ for $P \in \mathcal{S}_{\rho}(\mathbb{R}):=\mathcal{S}_{\rho} \cap \mathcal{E}(\mathbb{R})$ and $\mathcal{S}_{\rho}(\mathbb{R}) \cap \mathcal{H}_{\rho} \subset F_{\rho}(0)$.

Lemma 5.5. For each $\rho>0, \mathcal{S}_{\rho}(\mathbb{R})$ is a Jordan arc in $\mathbb{R}_{+}^{2}$.

Proof. Since $\mathcal{S}_{\rho}(\mathbb{R})$ intersects each stretching ray exactly at one point, it has neither self-intersection point nor closed loop component. On the other hand, it is a level curve of the function $H(P)$, which is equal to a real analytic function $h_{P}(-\sqrt{A})$ by Lemma 3.3. Hence it has no isolated point. In fact, its isolated point must be a local maximum or minimum point of $H(P)$ and for nearby $\rho^{\prime}, \mathcal{S}_{\rho^{\prime}}(\mathbb{R})$ must have a loop component; a contradiction. Thus, each component of $\mathcal{S}_{\rho}(\mathbb{R})$ can be continued to the $A$-axis or the $B$-axis. Then it must be a connected arc without self-intersection, hence a Jordan arc.

From the construction of the homeomorphism between $\mathcal{E}_{1} \cap F_{\rho}(\theta)$ and a single point or a copy of the Mandelbrot set in [B-H2], we have

Proposition 5.6. Each connected component of $\mathcal{S}_{\rho}(\mathbb{R}) \cap \mathcal{E}_{1}(\mathbb{R})$ is either a single point or a homeomorphic copy of the real locus of the Mandelbrot set.

Proposition 5.7. For any point $P_{0}=\left(A_{0}, B_{0}\right) \in \operatorname{Preper}_{(1) 1} \cap \partial \mathcal{C}(\mathbb{R})$, there is a stretching ray landing at $\left(A_{0}, B_{0}\right)$. Moreover, if there is no $k(\in \mathbb{N})$ satisfying $P_{0}^{k}\left(\sqrt{A_{0}}\right)=-\sqrt{A_{0}}$, i.e., just except the case treated in Proposition 4.3, there is a unique stretching ray landing at $\left(A_{0}, B_{0}\right)$. 
Proof. By the density of hyperbolic maps in $\operatorname{Preper}_{(1) 1} \cap \partial \mathcal{C}(\mathbb{R})$, there exist two sequences $\left(A_{n}, B_{n}\right),\left(A_{n}^{\prime}, B_{n}^{\prime}\right)$ of hyperbolic parameters on $\operatorname{Preper}_{(1) 1}$ converging to $\left(A_{0}, B_{0}\right)$ and satisfying $A_{n}<A_{0}<A_{n}^{\prime}$. From Proposition 5.3 there exist stretching rays $R_{n}, R_{n}^{\prime}$ landing at $\left(A_{n}, B_{n}\right),\left(A_{n}^{\prime}, B_{n}^{\prime}\right)$ respectively. Let $S_{n}$ be the closed region bounded by $R_{n}, R_{n}^{\prime}$ and the subarc $\left\{(A, B) \in \operatorname{Preper}_{(1) 1} \mid A_{n} \leq A \leq A_{n}^{\prime}\right\}$. Then $S=\bigcap_{n>1} S_{n}$ is a closed sector with vertex $\left(A_{0}, B_{0}\right)$ and the stretching ray $R(P)$ through $P \in S \backslash\left\{\left(A_{0}, B_{0}\right)\right\}$ must land at $\left(A_{0}, B_{0}\right)$.

Next, we assume $\left(A_{0}, B_{0}\right)$ satisfies no critical orbit relation of the form $P_{0}^{k}\left(\sqrt{A_{0}}\right)$ $=-\sqrt{A_{0}}$ and we show only one ray lands there. Suppose two rays land there. Then the open sector region $U$ bounded by these two rays is a subset of $\mathcal{E}_{1}(\mathbb{R})$ by Proposition 4.3. Now we use the notations prepared above. It is easy to see that $U \cap \mathcal{S}_{\rho}(\mathbb{R}) \subset F_{\rho}(0)$. Since $U \cap \mathcal{S}_{\rho}(\mathbb{R})$ is open in $\mathcal{S}_{\rho}(\mathbb{R})$, by Proposition 5.6 it must include an open subset of the copy of the real slice of the Mandelbrot set. Again by the density of hyperbolicity, it includes a subarc consisting of hyperbolic maps. This contradicts Proposition 5.1. Thus $\left(A_{0}, B_{0}\right)$ is the landing point of a unique stretching ray.

Summarizing the previous results, we have the following result of the landing problem of stretching rays in $\mathcal{R}_{2}$.

Theorem 5.8. For any $P \in \mathcal{E}_{1}(\mathbb{R}), R(P)$ lands at a point on Preper $_{(1) 1}$. Conversely, any $P_{0}=\left(A_{0}, B_{0}\right) \in$ Preper $_{(1) 1}$ is the landing point of a stretching ray. More precisely, if there is $k \in \mathbb{N}$ satisfying $P_{0}^{k}\left(\sqrt{A_{0}}\right)=-\sqrt{A_{0}}$, there are two stretching rays in $\mathcal{E}_{1}(\mathbb{R})$ landing at $P_{0}$ which form the boundary of a connected component of $\mathcal{E}_{2}(\mathbb{R}) \cap \mathcal{R}_{2}$. On the other hand, if there are no such critical orbit relations, there is a unique stretching ray in $\mathcal{E}_{1}(\mathbb{R})$ landing at $P_{0}$.

Question 5.9. For $P \in \mathcal{E}_{1}(\mathbb{R})$, let $K_{0}$ be the connected component of $K(P)$ containing $\sqrt{A}$, and denote $V_{P}=\left\{z \in \mathbb{C} \mid h_{P}(z)<H(P)\right\}$. Let $V_{P}^{k}$ be the connected component of $P^{-k}\left(V_{P}\right)$ containing $K_{0}$. Then Branner and Hubbard [B-H2] showed that $K(P)$ is a Cantor set if and only if $K_{0}$ is non-periodic under $P$. If $K_{0}$ is $k$-periodic, then $\left.P^{k}\right|_{V_{P}^{k}}: V_{P}^{k} \rightarrow V_{P}$ is a polynomial-like map of degree two, which is hybrid equivalent to a quadratic polynomial $p_{c}(z)=z^{2}+c$. Since stretching gives a hybrid equivalence, the parameter $c$ is constant on the stretching ray $R(P)$. On the other hand, from the previous argument we know that $R(P)$ lands at a point $P^{\prime} \in \operatorname{Preper}_{(1) 1} \cap \partial \mathcal{C}(\mathbb{R})$ which can be constructed from a quadratic polynomial $p_{c^{\prime}}(z)=z^{2}+c^{\prime}$ by the surgery in $\mathrm{B}-\mathrm{D}$. Does $c$ relate to $c^{\prime}$ ?

\section{LANDING AND NON-LANDING OF STRETCHING RAYS IN $\mathcal{R}_{0}$}

In this section, we consider landing and non-landing properties of stretching rays in $\mathcal{R}_{0}$. Here is the main theorem of this article.

Theorem 6.1. Suppose $P \in \mathcal{R}_{0}$ and $\eta(P)$ is not integral. Then the stretching ray $R(P)$ does not land at any point on $\operatorname{Per}_{1}(1) \cap \partial \mathcal{C}(\mathbb{R})$. That is, its accumulation set $I(P)$ is a non-trivial arc on $\operatorname{Per}_{1}(1) \cap \partial \mathcal{C}(\mathbb{R})$. If $\eta(P)$ is integral, then $R(P)$ lands at some point on $\operatorname{Per}_{1}(1) \cap \partial \mathcal{C}(\mathbb{R})$.

This section is devoted to the proof of this theorem. We give an outline of the proof here. In Subsection 6.1, we show that any stretching ray $R(P)$ with integral Böttcher vector $\eta(P)$ lands on $\operatorname{Per}_{1}(1) \cap \partial \mathcal{C}(\mathbb{R})$. 
The proof of the non-landing part is shown by contradiction. In Subsection 6.2 we define the Fatou vector on $\operatorname{Per}_{1}(1)$. By a qc-deformation of this vector, we get a parametrization of $\operatorname{Per}_{1}(1)$ by the Fatou vector. We are interested in the relation between the Böttcher vector $\eta(P)$ of a ray $R(P)$ and the Fatou vector $\tau(Q)$ of its accumulation point $Q \in \operatorname{Per}_{1}(1) \cap \partial \mathcal{C}(\mathbb{R})$. To study this, in Subsection 6.3. we define the Böttcher vector $\eta(Q, \sigma)$ also for $Q \in \operatorname{Per}_{1}(1) \cap \partial \mathcal{C}(\mathbb{R})$ by means of the Lavaurs map $g_{Q, \tilde{\sigma}}$, and partially characterize the accumulation set of each ray. Its complete characterization will be given in Section 7 by a more refined analysis.

Suppose a ray $R(P)$ lands at some $Q \in \operatorname{Per}_{1}(1) \cap \partial \mathcal{C}(\mathbb{R})$. Then we show that $\eta(P)$ coincides $\tau(Q)$. If $\eta(P)$ is irrational, we show $J(Q)$ is a real analytic curve, which is a contradiction. In Subsection 6.4, we consider the case where the Böttcher vector is non-integral rational. Then we need a stronger geometric property of $J(Q)$ to get a contradiction. That is, we essentially use the fact that $J(Q)$ is cusp at each point on the backward orbit of the parabolic fixed point $\beta_{Q}$.

6.1. Stretching rays with integral Böttcher vectors. First we shall show the stretching rays on which certain critical orbit relations hold actually land at points also having the same critical orbit relations. Note that $\operatorname{Per}_{1}(1) \cap \partial \mathcal{C}(\mathbb{R})$ is parametrized by $A\left(0<A \leq \frac{1}{9}\right)$ and $Q \in \operatorname{Per}_{1}(1) \cap \partial \mathcal{C}(\mathbb{R})$ can be written as $Q(z)=Q_{A}(z)=z^{3}-3 A z+2(A+1 / 3)^{3 / 2}$. For $Q \in \operatorname{Per}_{1}(1) \cap \partial \mathcal{C}(\mathbb{R})$ and $k \geq 0$, put $g_{k}(A):=Q(-\sqrt{A})-Q^{k+1}(\sqrt{A})$.

Lemma 6.2. $g_{k}$ is a monotone increasing function on $[0,1 / 9]$.

Proof. This is true for $k=0$ since $g_{0}(A)=4 A \sqrt{A}$. Suppose $k \geq 1$. By a direct calculation, we have

$$
\begin{aligned}
g_{k}^{\prime}(A) & =3\left(Q^{k}(\sqrt{A})+\sqrt{A}\right)-3\left(Q^{k}(\sqrt{A})^{2}-A\right) d Q^{k}(\sqrt{A}) / d A \\
& =3\left(Q^{k}(\sqrt{A})+\sqrt{A}\right)\left\{1-\left(Q^{k}(\sqrt{A})-\sqrt{A}\right) d Q^{k}(\sqrt{A}) / d A\right\} .
\end{aligned}
$$

Since $Q^{k}(\sqrt{A})>\sqrt{A}$, we have only to show

$$
d Q^{k}(\sqrt{A}) / d A<\frac{1}{Q^{k}(\sqrt{A})-\sqrt{A}}, \quad 0 \leq A \leq 1 / 9 .
$$

We do this by induction on $k$. For $k=1$,

$$
\begin{aligned}
& \frac{1}{d Q(\sqrt{A}) / d A}-(Q(\sqrt{A})-\sqrt{A}) \\
& \quad=\frac{1}{3 \sqrt{A+1 / 3}-3 \sqrt{A}}-\left\{2(A+1 / 3)^{3 / 2}-2 A^{3 / 2}-\sqrt{A}\right\} \\
& \quad=\sqrt{A+1 / 3}+\sqrt{A}-\left\{2(A+1 / 3)^{3 / 2}-2 A^{3 / 2}-\sqrt{A}\right\} \\
& \quad=\sqrt{A+1 / 3}\{1-2(A+1 / 3)\}+2 \sqrt{A}(A+1)>0,
\end{aligned}
$$


and the conclusion is true. Suppose it is true for $k$. By the induction hypothesis,

$$
\begin{aligned}
\frac{d Q^{k+1}(\sqrt{A})}{d A} & =3\left(\sqrt{A+1 / 3}-Q^{k}(\sqrt{A})\right)+3\left(Q^{k}(\sqrt{A})^{2}-A\right) \frac{d Q^{k}(\sqrt{A})}{d A} \\
& <3\left(\sqrt{A+1 / 3}-Q^{k}(\sqrt{A})\right)+3\left(Q^{k}(\sqrt{A})+\sqrt{A}\right) \\
& =\frac{1}{\sqrt{A+1 / 3}-\sqrt{A}} \\
& =\frac{1}{\beta_{P}-\sqrt{A}} \\
& <\frac{1}{Q^{k+1}(\sqrt{A})-\sqrt{A}} .
\end{aligned}
$$

Hence the conclusion also holds for $k+1$. This completes the proof.

Since $g_{k}(0)=Q_{0}(0)-Q_{0}^{k+1}(0) \leq 0$ and $g_{k}(1 / 9)=Q_{1 / 9}(-1 / 3)-Q_{1 / 9}^{k+1}(1 / 3)=$ $\beta_{Q_{1 / 9}}-Q_{1 / 9}^{k+1}(1 / 3)>0, g_{k}$ has a unique zero $A_{k}$ in $[0,1 / 9)$. Since $Q^{k}(\sqrt{A})<$ $Q^{k+1}(\sqrt{A})$, it follows that $A_{k-1}<A_{k}$.

Proposition 6.3. For any integer $k \geq 0$, there is a unique stretching ray $R(k)$ in $\mathcal{R}_{0}$ with Böttcher vector $k$, landing at $Q_{A_{k}} \in \operatorname{Per}_{1}(1) \cap \partial \mathcal{C}(\mathbb{R}) . R(k)$ is expressed by the critical orbit relation $P(-\sqrt{A})-P^{k+1}(\sqrt{A})=0$.

Proof. The above estimate holds also for small perturbation $Q_{A, \epsilon}(z)=z^{3}-3 A z+$ $2(A+1 / 3)^{3 / 2}+\epsilon, \epsilon>0$ above $\operatorname{Per}_{1}(1) \cap \partial \mathcal{C}(\mathbb{R})$. Thus there exists a unique real algebraic curve $P(-\sqrt{A})-P^{k+1}(\sqrt{A})=0$ through the point $Q_{A_{k}} \in \operatorname{Per}_{1}(1) \cap \partial \mathcal{C}(\mathbb{R})$. Since this critical orbit relation is preserved under stretching, it forms a stretching ray and is real analytic. Then it must land at some point on $\operatorname{Per}_{1}(1) \cap \partial \mathcal{C}(\mathbb{R})$, since $\mathrm{Per}_{1}(1)$ is also real analytic.

Although $R(0)$ lies on the $B$-axis, it also defines a stretching ray. By continuity of the Böttcher vector map, we have the following.

Corollary 6.4. For any $\eta>0$, there exists, in $\mathcal{R}_{0}$, at least one stretching ray with Böttcher vector $\eta$.

6.2. Parametrization of $\operatorname{Per}_{1}(1)$ by Fatou vectors. Next we consider the stretching rays whose Böttcher vectors are not integers. For $Q \in \operatorname{Per}_{1}(1) \cap \partial \mathcal{C}(\mathbb{R})$, the immediate basin $\mathcal{B}_{Q}$ of the parabolic fixed point $\beta_{Q}$ contains both critical points $\pm \sqrt{A}$. Therefore $\mathcal{B}_{Q}$ is completely invariant. Since $J_{Q}=\partial \mathcal{B}_{Q}$ is locally connected, it is a Jordan curve ([C-G, Example after Theorem 4.3 in Chapter 5]).

Let $\phi_{Q,-}$ and $\phi_{Q,+}$ be the attracting and repelling Fatou coordinates respectively ([D, La and $\underline{\mathrm{Sh}}])$. Originally, they are defined only on the attracting and repelling petals $\Omega_{Q,-}$ and $\Omega_{Q,+}$ respectively and satisfy the functional equation: $\phi_{Q, \pm} \circ Q=T_{1} \circ \phi_{Q, \pm}$, where $T_{1}(w)=w+1$ is the translation by one. They can be continued analytically by this relation. Especially $\phi_{Q,-}$ is continued to the basin $\mathcal{B}_{Q}$. Fatou coordinates are unique up to additive constants. Therefore we normalize them with the conditions $\phi_{Q, \pm}\left(c_{ \pm}(Q)\right)=0$, where $c_{ \pm}(Q)$ is a real-valued continuous function contained in the domain of $\phi_{Q, \pm}$ respectively. In particular, they satisfy $c_{-}(Q)<\beta_{Q}<c_{+}(Q)$ for all $Q \in \operatorname{Per}_{1}(1) \cap \partial \mathcal{C}(\mathbb{R})$. Then $\phi_{Q, \pm}$ are determined uniquely, depend continuously on $Q$ and commute with complex conjugation; especially real valued on the real axis. Actually we will take $c_{-}(Q)=\sqrt{A}$ or $Q(-\sqrt{A})$ 
and $c_{+}(Q)=c_{+}$, a fixed constant. We define the Fatou vector $\tau(Q)$ of $Q$ by $\tau(Q):=\phi_{Q,-}(-\sqrt{A})-\phi_{Q,-}(\sqrt{A})$, the difference of the critical points in the attracting Fatou coordinate. Note that this vector $\tau(Q)$ does not depend on the choice of the Fatou coordinates.

Proposition 6.5. The Fatou vector gives a real analytic parametrization of $\operatorname{Per}_{1}(1)$ $\cap \partial \mathcal{C}(\mathbb{R})$.

Proof. First we show that the Fatou vector map $Q \mapsto \tau(Q)$ has a local real analytic inverse in each connected component of $\mathbb{R}_{+} \backslash \mathbb{N}$ by virtue of qc-deformation. Suppose $k<\tau_{0}=\tau\left(Q_{0}\right)<k+1$. Take any $\tau \in(k, k+1)$. Consider the piecewise affine map $S_{\tau}(x+y i)=s_{\tau}(x)+y i$, where

$$
s_{\tau}(x):=\left\{\begin{array}{l}
k+\frac{\tau-k}{\tau_{0}-k}(x-k) \text { if } k \leq x \leq \tau_{0}, \\
k+1-\frac{k+1-\tau}{k+1-\tau_{0}}(k+1-x) \text { if } \tau_{0} \leq x \leq k+1 .
\end{array}\right.
$$

It is easy to see that $S_{\tau}$ is a qc-map from the strip $V=\{k \leq \operatorname{Re} w \leq k+1\}$ onto itself, identity on its boundary, and satisfies $S_{\tau}\left(\tau_{0}\right)=\tau$. We extend it to the whole plane by the functional equation $S_{\tau} \circ T_{1}=T_{1} \circ S_{\tau}$.

Let $\left.\sigma_{\tau}\right|_{V_{0}}=\left(S_{\tau} \circ \phi_{Q_{0},-}\right)^{*} \sigma_{0}$ be a deformation of the standard complex structure $\sigma_{0}$ in the fundamental region $V_{0}=\phi_{Q_{0},-}^{-1}(V)$ of $Q_{0}$. In each connected component of $Q_{0}^{-n}\left(V_{0}\right)$, we set $\sigma_{\tau}=\left(Q_{0}^{n}\right)^{*}\left(\left.\sigma_{\tau}\right|_{V_{0}}\right)$. Then we get a $Q_{0}$-invariant complex structure $\sigma_{\tau}$ in $\mathcal{B}_{Q_{0}}$. If we set $\sigma_{\tau}=\sigma_{0}$ outside the filled-in Julia set $K_{Q_{0}}, \sigma_{\tau}$ is $Q_{0}$-invariant on $\mathbb{C}$ and depends real analytically on $\tau$. Let $\xi_{\tau}$ be the integrating qc-map of $\sigma_{\tau}$ so that $Q_{\tau}=\xi_{\tau} \circ Q_{0} \circ \xi_{\tau}^{-1} \in \operatorname{Per}_{1}(1) \cap \partial \mathcal{C}(\mathbb{R})$.

Note that $S_{\tau} \circ \phi_{Q_{0},-} \circ \xi_{\tau}^{-1}$ is a Fatou coordinate of $Q_{\tau}$. Then $\tau\left(Q_{\tau}\right)=\tau$. This gives a local real analytic inverse of the Fatou vector map $\tau$.

The above argument does not work when $\tau_{0}=k \in \mathbb{N}$. In this case, critical orbit relation $Q_{0}^{k+1}(\sqrt{A})=Q_{0}(-\sqrt{A})$ holds and $Q_{0}$ cannot be qc-conjugate to any maps nearby. We do qc-surgery instead of qc-deformation, because we have to change the dynamics.

We normalize the attracting Fatou coordinates by $\phi_{Q_{0},-}\left(Q_{0}(-\sqrt{A})\right)=0$. Note that this does not change the Fatou vectors. Then $\phi_{Q_{0},-}\left(Q_{0}(\sqrt{A})\right)=-k$. Take a small open neighborhood $U$ of 0 in the attracting Fatou coordinate and let $s_{\tau}$ : $U \rightarrow U$ be a qc-map, identity on $\partial U, s_{0}=i d$ and $s_{\tau}(0)=\tau \in(-\epsilon, \epsilon)$ for some small $\epsilon>0$. Take an open neighborhood $V$ of $Q_{0}^{k}(\sqrt{A})$ so that $U \subset T_{1}\left(\phi_{Q_{0},-}(V)\right)$ and put $U^{\prime}=T_{1}^{-1}(U), V^{\prime}=\phi_{Q_{0},-}^{-1}\left(U^{\prime}\right) \cap V$. We define a qc-map $S_{\tau}$ by

$$
S_{\tau}:=\left\{\begin{array}{l}
s_{\tau} \circ T_{1} \text { on } U^{\prime} \\
T_{1} \text { elsewhere. }
\end{array}\right.
$$

Then the map $R_{\tau}$, defined by

$$
R_{\tau}:=\left\{\begin{array}{l}
\phi_{Q_{0},-}^{-1} \circ S_{\tau} \circ \phi_{Q_{0},-} \text { on } V^{\prime}, \\
Q_{0} \text { elsewhere. }
\end{array}\right.
$$

is a quasi-regular map on $\mathcal{B}_{Q_{0}}$ depending real analytically on $\tau$.

Let $V_{0}$ be a fundamental region of $Q_{0}$ containing $V$ and set $\sigma_{\tau}=\phi_{Q_{0},-}^{*} s_{\tau}^{*} \sigma_{0}$ and $\sigma_{\tau}=\sigma_{0}$ on $V_{0}$ and $R_{\tau}\left(V_{0}\right)$ respectively, and set $\sigma_{\tau}=\left(R_{\tau}^{n}\right)^{*} \sigma_{\tau}$ in each connected component of $R_{\tau}^{-n}\left(V_{0}\right)$.

Then we get an $R_{\tau}$-invariant complex structure $\sigma_{\tau}$ on $\mathcal{B}_{Q_{0}}$. Put $\sigma_{\tau}=\sigma_{0}$ outside $K_{Q_{0}}$. Let $\xi_{\tau}$ be its integrating qc-map such that $Q_{\tau}^{\prime}=\xi_{\tau} \circ R_{\tau} \circ \xi_{\tau}^{-1} \in \operatorname{Per}_{1}(1) \cap$ 
$\partial \mathcal{C}(\mathbb{R})$. Then $Q_{\tau}^{\prime}$ depends real analytically on $\tau, Q_{0}^{\prime}=Q_{0}$ and $\tau\left(Q_{\tau}^{\prime}\right)=k+\tau$. Thus we obtain a real analytic local parametrization $Q_{\tau}^{\prime}$ of $\operatorname{Per}_{1}(1) \cap \partial \mathcal{C}(\mathbb{R})$ around $Q_{0}^{\prime}=Q_{0}$ with $\tau\left(Q_{0}^{\prime}\right)=k$. This completes the proof.

The Fatou vector corresponds to $0<\tau\left(Q_{A}\right)<\infty$ on $0<A<1 / 9$. In terms of Fatou vectors, Proposition 6.3 can be expressed as follows.

Proposition 6.6. The stretching ray $R(k)$ in $\mathcal{R}_{0}$ with Böttcher vector $k \in \mathbb{N}$ lands at a map $Q \in \operatorname{Per}_{1}(1) \cap \partial \mathcal{C}(\mathbb{R})$ with $\tau(Q)=k$. Conversely, any $Q \in \operatorname{Per}_{1}(1) \cap \partial \mathcal{C}(\mathbb{R})$ with $\tau(Q)=k \in \mathbb{N}$, is the landing point of the ray $R(k)$.

The "limit" of $R(k)$ as $k \rightarrow \infty$ is also a stretching ray

$$
R(\infty): B=4(A+1 / 3)^{3}, \quad A>1 / 9,
$$

which is equal to $\operatorname{Per}_{1}(1) \cap\left(\mathbb{R}_{+}^{2} \backslash \mathcal{C}(\mathbb{R})\right)$. It lands at $\left(A_{\infty}, B_{\infty}\right)=\left(1 / 9,4^{4} / 9^{3}\right)$.

6.3. Stretching rays with irrational Böttcher vectors. In the following two subsections, we show the non-landing part of Theorem 6.1 By qc-deformation of Böttcher vectors along $\mathcal{S}_{\rho} \cap \mathcal{R}_{0}$ just like the proof of Proposition [6.5, we can show the unique existence of the stretching ray in $\mathcal{R}_{0}$ with a given Böttcher vector $\eta>0$. We denote it by $R(\eta)$.

The proof is an application of the parabolic implosion analysis; see [D, $\mathrm{La}$, $\underline{\mathrm{Sh}}$ or W]. First we prepare some notions from the theory of parabolic implosion. The following lemma, a consequence of a direct calculation, assures the existence of the Fatou coordinates for $Q_{A, \epsilon}$.

Lemma 6.7. Let $\beta_{Q_{A, \epsilon}}^{ \pm}$be the fixed points of $Q_{A, \epsilon}$ bifurcating from $\beta_{Q_{A}}$ and let $\rho_{ \pm}(\epsilon)$ be their multipliers. Then we have

$$
\begin{aligned}
& \beta_{Q_{A, \epsilon}}^{ \pm}=\sqrt{A+1 / 3} \pm i \sqrt{\frac{\epsilon}{3 A+1}}+\frac{\epsilon}{18 A+6}+O\left(\epsilon^{3 / 2}\right), \\
& \rho_{ \pm}(\epsilon)=1 \pm 2 i(A+1 / 3)^{1 / 4} \sqrt{3 \epsilon}-\frac{2 \epsilon}{3 \sqrt{A+1 / 3}}+O\left(\epsilon^{3 / 2}\right) .
\end{aligned}
$$

So, let $\phi_{P, \pm}$ be the Fatou coordinates of $P \in \mathcal{R}_{0}$, close to $\operatorname{Per}_{1}(1) \cap \partial \mathcal{C}(\mathbb{R})$, normalized by $\phi_{P, \pm}\left(c_{ \pm}(P)\right)=0$. They are continuous up to $\operatorname{Per}_{1}(1) \cap \partial \mathcal{C}(\mathbb{R})$. After perturbation, the gate between two fixed points $\beta_{P}^{ \pm}$is open and the incoming Fatou coordinate $\phi_{P,-}$ can be regarded also as an outgoing Fatou coordinate $\phi_{P,+}$ and vice versa. Thus $\phi_{P,+}$ and $\phi_{P,-}$ differ only by an additive constant. We call this difference $\tilde{\sigma}(P)=\phi_{P,+}(z)-\phi_{P,-}(z)$ the lifted phase and its class $\sigma(P)=[\tilde{\sigma}(P)]$ in $\mathbb{C} / \mathbb{Z}$ the phase of $P$. Since all mappings are symmetric with respect to the real axis, the lifted phase is always real. Roughly speaking, minus the lifted phase is the time needed for the orbits of $P$ to pass through the gate.

Lemma 6.8 (cf. [D, Corollary 17.4]). The lifted phase $\tilde{\sigma}\left(P_{s}\right)$ tends to $-\infty$ as $s \rightarrow 0$ on each stretching ray.

Proof. Put $c_{ \pm}=c_{ \pm}\left(P_{s}\right)$. Then for any $s$, there exists an $n=n_{s}$ such that $c_{+} \leq$ $P_{s}^{n}\left(c_{-}\right)<P_{s}\left(c_{+}\right)$. Since

$$
0=\phi_{P_{s},+}\left(c_{+}\right) \leq \phi_{P_{s},+}\left(P_{s}^{n}\left(c_{-}\right)\right)=\phi_{P_{s},+}\left(c_{-}\right)+n<\phi_{P_{s},+}\left(P_{s}\left(c_{+}\right)\right)=1,
$$

it follows that $-n \leq \tilde{\sigma}\left(P_{s}\right)=\phi_{P_{s},+}\left(c_{-}\right)<-n+1$. Suppose $\tilde{\sigma}\left(P_{s}\right)$ does not tend to $-\infty$ as $s \rightarrow 0$. Then there exists a $k$ and a sequence $P_{n} \in R(P)$ such that 
$\tilde{\sigma}\left(P_{n}\right) \geq-k$. This implies $P_{n}^{k}\left(c_{-}\right) \geq c_{+}$. We may assume $P_{n}$ tends to some $Q \in \operatorname{Per}_{1}(1) \cap \partial \mathcal{C}(\mathbb{R})$ by taking a subsequence if necessary. Then it follows that $Q^{k}\left(c_{-}\right) \geq c_{+}$, which is a contradiction. This completes the proof.

We also define, for $Q \in \operatorname{Per}_{1}(1) \cap \partial \mathcal{C}(\mathbb{R})$ and for $\tilde{\sigma} \in \mathbb{C}$, the Lavaurs map $g_{Q, \tilde{\sigma}}$ : $\mathcal{B}_{Q} \rightarrow \mathbb{C}$ with lifted phase $\tilde{\sigma}$ by $g_{Q, \tilde{\sigma}}:=\phi_{Q,+}^{-1} \circ T_{\tilde{\sigma}} \circ \phi_{Q,-}$, where $T_{\tilde{\sigma}}(w)=w+\tilde{\sigma}$. The following is a fundamental fact for $g_{Q, \tilde{\sigma}}$. (See [D], Proposition 18.2, for example.)

Lemma 6.9. Suppose $P_{n} \in \mathcal{R}_{0}$ satisfies $P_{n} \rightarrow Q \in \operatorname{Per}_{1}(1) \cap \partial \mathcal{C}(\mathbb{R})$ and $\sigma\left(P_{n}\right) \rightarrow$ $\sigma \in \mathbb{C} / \mathbb{Z}$. Let $\tilde{\sigma}$ be any lift of $\sigma$. If we take $N_{n} \rightarrow \infty$ satisfying $N_{n}+\tilde{\sigma}\left(P_{n}\right) \rightarrow \tilde{\sigma}$, then $P_{n}^{N_{n}} \rightarrow g_{Q, \tilde{\sigma}}$ locally uniformly on $\mathcal{B}_{Q}$.

Proof. By the continuity of Fatou coordinates up to $\operatorname{Per}_{1}(1) \cap \partial \mathcal{C}(\mathbb{R})$, we have

$$
\begin{aligned}
P_{n}^{N_{n}} & =\phi_{P_{n},+}^{-1} \circ\left(\phi_{P_{n},+} \circ P_{n}^{N_{n}} \circ \phi_{P_{n},-}^{-1}\right) \circ \phi_{P_{n},-} \\
& =\phi_{P_{n},+}^{-1} \circ\left(T_{N_{n}} \circ \phi_{P_{n},+} \circ \phi_{P_{n},-}^{-1}\right) \circ \phi_{P_{n},-} \\
& =\phi_{P_{n},+}^{-1} \circ\left(T_{N_{n}} \circ T_{\tilde{\sigma}\left(P_{n}\right)}\right) \circ \phi_{P_{n},-} \\
& =\phi_{P_{n},+}^{-1} \circ T_{N_{n}+\tilde{\sigma}\left(P_{n}\right)} \circ \phi_{P_{n},-} \\
& \rightarrow \phi_{Q,+}^{-1} \circ T_{\tilde{\sigma}} \circ \phi_{Q,-} \\
& =g_{Q, \tilde{\sigma}}
\end{aligned}
$$

locally uniformly on $\mathcal{B}_{Q}$. This completes the proof.

Since, in our case, $K(Q)$ is symmetric with respect to the real axis, connected and locally connected, its image in the repelling Fatou coordinate does not intersect the real axis. Then it follows that $g_{Q, \tilde{\sigma}}( \pm \sqrt{A}) \in \mathbb{C}-K(Q)$ for real $\tilde{\sigma}$. Hence, we can define the Böttcher vector $\eta(Q, \sigma)$ with phase $\sigma \in \mathbb{R} / \mathbb{Z}$ also for $Q \in \operatorname{Per}_{1}(1) \cap \partial \mathcal{C}(\mathbb{R})$ :

$$
\eta(Q, \sigma):=\zeta_{Q}\left(g_{Q, \tilde{\sigma}}(-\sqrt{A})\right)-\zeta_{Q}\left(g_{Q, \tilde{\sigma}}(\sqrt{A})\right) .
$$

It depends only on the phase and not on the choice of lifted phase because $\zeta_{Q}$ satisfies $\zeta_{Q} \circ Q=T_{1} \circ \zeta_{Q}$. Note that this definition depends on the choice of Fatou coordinates. In fact, if we add some constants to them, this changes the phase. This causes some difficulty in the next section. We define the $\sigma$-impression $I_{\eta}(\sigma)$ of $R(\eta)$ by the set of points $Q \in \operatorname{Per}_{1}(1) \cap \partial \mathcal{C}(\mathbb{R})$ such that there exists $P_{n} \in R(\eta)$ satisfying $P_{n} \rightarrow Q$ and $\sigma\left(P_{n}\right) \rightarrow \sigma$. Apparently the accumulation set $I(\eta)$ of $R(\eta)$ is the union of all $I_{\eta}(\sigma), \sigma \in \mathbb{R} / \mathbb{Z}$.

Lemma 6.10. Suppose $P_{n} \in \mathcal{R}_{0}$ satisfies $P_{n} \rightarrow Q \in \operatorname{Per}_{1}(1) \cap \partial \mathcal{C}(\mathbb{R})$ and $\sigma\left(P_{n}\right) \rightarrow$ $\sigma \in \mathbb{C} / \mathbb{Z}$. Then $\eta\left(P_{n}\right) \rightarrow \eta(Q, \sigma)$. Consequently, we have $I_{\eta}(\sigma) \subset\left\{Q \in \operatorname{Per}_{1}(1) \cap\right.$ $\partial \mathcal{C}(\mathbb{R}) \mid \eta(Q, \sigma)=\eta\}$.

Proof. By Lemma 6.9, for any lift $\tilde{\sigma}$ of $\sigma$, there exists a sequence $N_{n} \rightarrow \infty$ such that $P_{n}^{N_{n}} \rightarrow g_{Q, \tilde{\sigma}}$ locally uniformly in $\mathcal{B}_{Q}$. Then it follows

$$
\begin{aligned}
\eta\left(P_{n}\right) & =\zeta_{P_{n}}\left(P_{n}\left(-\sqrt{A\left(P_{n}\right)}\right)\right)-\zeta_{P_{n}}\left(P_{n}\left(\sqrt{A\left(P_{n}\right)}\right)\right) \\
& =\zeta_{P_{n}}\left(P_{n}^{N_{n}}\left(-\sqrt{A\left(P_{n}\right)}\right)\right)-\zeta_{P_{n}}\left(P_{n}^{N_{n}}\left(\sqrt{A\left(P_{n}\right)}\right)\right) \\
& \rightarrow \zeta_{Q}\left(g_{Q, \tilde{\sigma}}(-\sqrt{A(Q)})\right)-\zeta_{Q}\left(g_{Q, \tilde{\sigma}}(\sqrt{A(Q)})\right) \\
& =\eta(Q, \sigma) .
\end{aligned}
$$

Here we use the fact that $\varphi_{P_{n}} \rightarrow \varphi_{Q}$ locally uniformly in $\mathbb{C}-K(Q)$. 


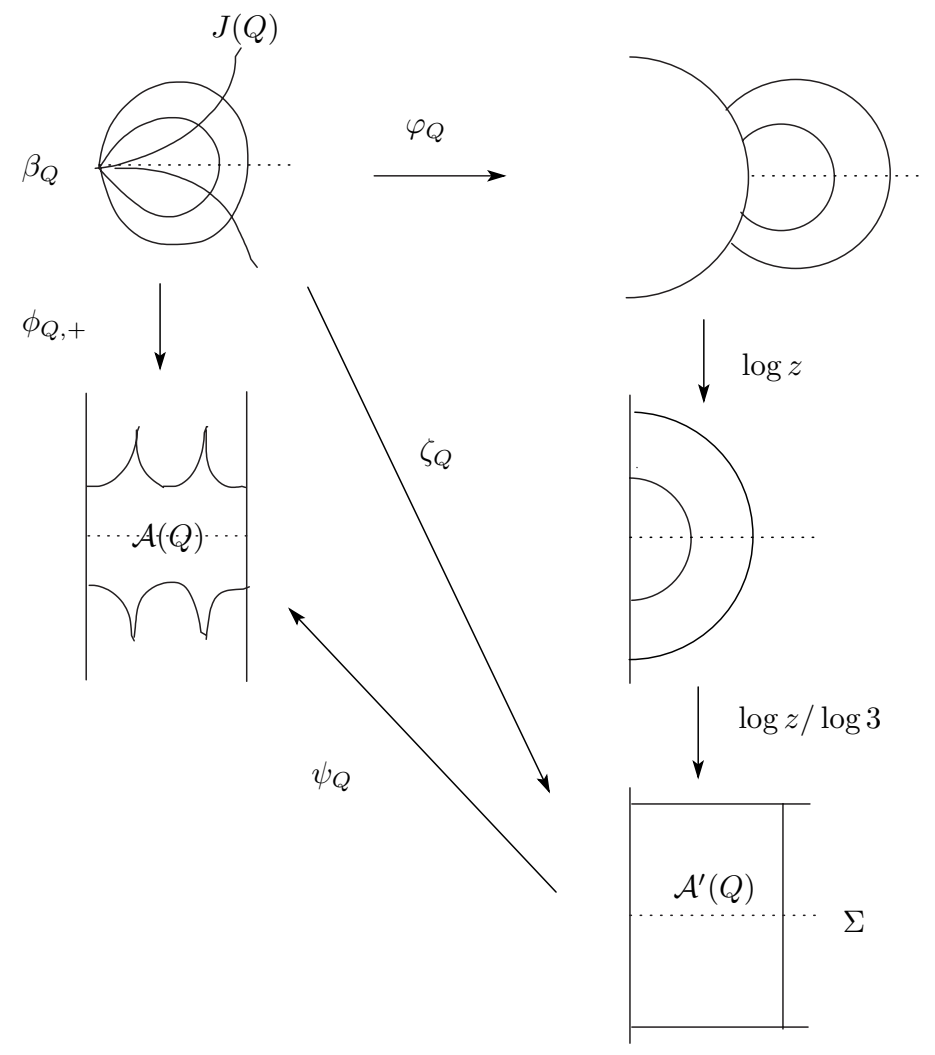

FiguRE 8. Annuli isomorphism $\psi_{Q}$ between $\mathcal{A}^{\prime}(Q)$ and $\mathcal{A}(Q)$

In the next section, we will prove the converse inclusion. Generally speaking, $\eta(Q, \sigma)$ depends on $\sigma$. But we have

Proposition 6.11. Suppose $R(\eta)$ lands at $Q \in \operatorname{Per}_{1}(1) \cap \partial \mathcal{C}(\mathbb{R})$. Then $\eta(Q, \sigma)$ is equal to $\eta$ for any $\sigma \in \mathbb{R} / \mathbb{Z}$. Especially, $\eta(Q, \sigma)$ is independent of $\sigma \in \mathbb{R} / \mathbb{Z}$.

Proof. Suppose $R(\eta)$ lands at $Q$. Then Lemma 6.8 says that, for any $\sigma \in \mathbb{R} / \mathbb{Z}$, there exists a sequence $P_{n} \in R(\eta)$ tending to $Q$ and satisfying $\sigma\left(P_{n}\right)=\sigma$. By Lemma 6.10, it follows that $\eta(Q, \sigma)=\lim _{n \rightarrow \infty} \eta\left(P_{n}\right)=\eta$ for any $\sigma$.

This proposition is key to the proof of Theorem 6.1. Let

$$
\mathcal{A}(Q)=\phi_{Q,+}\left(\Omega_{Q,+}-K(Q)\right) / T_{1}
$$

be the annulus in the repelling Ecalle cylinder $\phi_{Q,+}\left(\Omega_{Q,+}\right) / T_{1}$, bounded by the images of the Julia set $J(Q)$. Note that $\zeta_{Q}$ maps $\Omega_{Q,+}-K(Q)$ conformally onto a left half region $\Sigma$ in the strip $\{|\operatorname{Im} \zeta|<\pi /(2 \log 3)\}$ and satisfies $\zeta_{Q} \circ Q(z)=\zeta_{Q}(z)+1$ there (the same functional equation as the Fatou coordinates). This induces a flat annulus $\mathcal{A}^{\prime}(Q)=\{\zeta \in \mathbb{C} / \mathbb{Z}|| \operatorname{Im} \zeta \mid<\pi /(2 \log 3)\}$ of modulus $\pi / \log 3$. Then the induced map $\psi_{Q}: \mathcal{A}^{\prime}(Q) \rightarrow \mathcal{A}(Q)$ of the map $\phi_{Q,+}{ }^{\circ} \zeta_{Q}^{-1}: \Sigma \rightarrow \Omega_{Q,+}-K(Q)$ gives a conformal equivalence between the annuli $\mathcal{A}^{\prime}(Q)$ and $\mathcal{A}(Q)$; see Figure 8 .

In terms of the Lavaurs map, the Fatou vector is also written by

$$
\tau(Q)=\phi_{Q,+}\left(g_{Q, \tilde{\sigma}}(-\sqrt{A})\right)-\phi_{Q,+}\left(g_{Q, \tilde{\sigma}}(\sqrt{A})\right),
$$


which easily follows from the definition. Now we can see the geometric meanings of $\tau(Q)$ and $\eta(Q, \sigma)$. That is, $\tau(Q)$ is the difference of $g_{Q, \tilde{\sigma}}( \pm \sqrt{A})$ in the repelling Fatou coordinate, while $\eta(Q, \sigma)$ is their difference in the $\zeta_{Q}$-coordinate.

Lemma 6.12. Suppose $R(\eta)$ lands at $Q \in \operatorname{Per}_{1}(1) \cap \partial \mathcal{C}(\mathbb{R})$. Then $\psi_{Q}(\zeta+[\eta])=$ $\psi_{Q}(\zeta)+[\tau(Q)]$ holds for $\zeta \in \mathcal{A}^{\prime}(Q)$. Especially it follows $\tau(Q)=\eta$.

Proof. Since $\psi_{Q}$ is conformal, we have only to show the relation on the equator $\mathbb{R} / \mathbb{Z}$. Note that $\tau(Q)$ does not depend on $\sigma$ while $\eta(Q, \sigma)$ generally depends on $\sigma$. However, Proposition 6.11 assures its independence of $\sigma$ if $R(\eta)$ lands at $Q$. If we change $\sigma$, the positions of $g_{\tilde{\sigma}}( \pm \sqrt{A})$ in the repelling Fatou coordinate are translated according to that change. Nevertheless, their difference in the $\zeta_{Q}$-coordinate does not change. We can take $\sigma$ arbitrarily. So we conclude that, if two points on the real axis of the repelling Fatou coordinate have difference $\tau(Q)$, then their images by $\zeta_{Q} \circ \phi_{Q,+}^{-1}$ always have difference $\eta$. Hence we have $\psi_{Q}^{-1}(w+[\tau(Q)])=\psi_{Q}^{-1}(w)+[\eta]$ on the equator of $\mathcal{A}(Q)$, which implies $\psi_{Q}(\zeta+[\eta])=\psi_{Q}(\zeta)+[\tau(Q)]$ on the equator of $\mathcal{A}^{\prime}(Q)$. Then $\psi_{Q}$ gives a real analytic conjugacy of the two rotations on the equators with rotation numbers $[\tau(Q)]$ and $[\eta]$. Hence $[\tau(Q)]=[\eta]$. Now, suppose $\tau(Q)$ and $\eta$ have distinct integral parts. Then, by Proposition 6.6] $R(\eta)$ and $Q$ are separated by a stretching ray with integral Böttcher vector and its land point, which contradicts the assumption. Thus we conclude $\tau(Q)=\eta$. This completes the proof.

Proof of Theorem 6.1 (irrational case). Note that we have not used the assumption that $\eta$ is non-integral so far. Suppose $\eta$ is irrational and $R(\eta)$ lands at some $Q \in \operatorname{Per}_{1}(1) \cap \partial \mathcal{C}(\mathbb{R})$. By using Lemma 6.12 recursively, we have $\psi_{Q}(\zeta+[n \eta])=$ $\psi_{Q}(\zeta)+[n \tau(Q)]$ for any $n \in \mathbb{Z}$. If $\eta$ is irrational, the set $\{[n \eta] \mid n \in \mathbb{Z}\}$ is dense in $\mathbb{R} / \mathbb{Z}$. Then $\mathcal{A}(Q)$ must also be a flat annulus, i.e., its boundary $\partial \mathcal{A}(Q)$ consists of two round circles. Hence, as a preimage of $\partial \mathcal{A}(Q)$ by the conformal map $\phi_{Q,+}$, $J(Q)$ is a real analytic curve. This leads to a contradiction; see Figure 8 In fact, let $w=\tau_{0}(z):=-\frac{1}{3 \beta_{Q}\left(z-\beta_{Q}\right)}$ be an approximate Fatou coordinate. Then

$$
F(w):=\tau_{0} \circ Q \circ \tau_{0}^{-1}(w)=w+1+O\left(\frac{1}{w}\right)
$$

has an attracting Fatou coordinate in the sector $S_{-}=\left\{w \in \mathbb{C} \mid \operatorname{Re}\left(w-b_{-}\right)>\right.$ $\left.-\left|\operatorname{Im}\left(w-b_{-}\right)\right|\right\}$for some $b_{-} \in \mathbb{R}$ (cf. [Sh] $)$. Thus the immediate basin of $\beta_{Q}$ contains, locally at $\beta_{Q}$, a sector region with angle $3 \pi / 2$. Then $J(Q)=\partial \mathcal{B}_{Q}$ cannot be smooth at $\beta_{Q}$, consequently at all its preimages densely distributed on $J(Q)$. Thus the case of irrational Böttcher vectors in Theorem 6.1 is proved.

Remark 6.13. The Fatou vector is defined also for complex cubic polynomials with parabolic cycles. In $[\mathrm{W}]$ she conjectured that no complex stretching ray accumulates at points in $\operatorname{Per}_{1}(1)$ with non-real Fatou vectors and gave examples of $Q$ and $\sigma$ with non-real Böttcher vector $\eta(Q, \sigma)$.

6.4. Stretching rays with rational Böttcher vectors. In this subsection, we consider the case of non-integral rational Böttcher vectors. We begin with a notion of the radial Julia set for a rational map $f$.

Definition 6.14 (cf. [Mc, Section 2]). The radial Julia set $J_{\text {rad }}(f)$ of a rational map $f$ is defined as follows. First, $p \in J(f)$ belongs to $J_{\text {rad }}(f, r)$ if for any $\epsilon>0$, 
there exists a neighborhood $U$ of $p$ and $n>0$ such that the diameter $\operatorname{diam}(U)$ of $U$ is less than $\epsilon$ and

$$
f^{n}: U \rightarrow B\left(f^{n}(p), r\right)
$$

is a homeomorphism. Then set

$$
J_{\text {rad }}(f)=\bigcup_{r>0} J_{\text {rad }}(f, r) .
$$

We call a rational map $f$ geometrically finite if every critical point on the Julia set $J(f)$ is preperiodic (cf. [Mc, Section 6]). Note that each map $Q \in \operatorname{Per}_{1}(1) \cap \partial \mathcal{C}(\mathbb{R})$ is geometrically finite. For a geometrically finite rational map, we can describe the complement of $J_{\text {rad }}(f)$ in $J(f)$.

Theorem 6.15 (cf. $\mathrm{Mc}$, Theorem 6.5]). Let $f$ be a geometrically finite rational map. Then the complement of $J_{\text {rad }}(f)$ in $J(f)$ consists exactly of the backward orbits of the parabolic periodic points and critical points in $J(f)$.

Lemma 6.16. For $p / q \in \mathbb{Q}_{+}-\mathbb{N}$, suppose $R(p / q)$ lands at some $Q \in \operatorname{Per}_{1}(1) \cap$ $\partial \mathcal{C}(\mathbb{R})$. Then $\tau(Q)=p / q$ and the image of $J(Q)$ in the repelling Fatou coordinate is invariant under the translation $T_{1 / q}: w \mapsto w+1 / q$.

Proof. We may assume that $p$ and $q$ are prime to each other. Then there exist $m, n \in \mathbb{Z}$ such that $m p+n q=1$. Since $1 / q \equiv(m p+n q) / q \equiv m p / q$ in $\mathbb{R} / \mathbb{Z}$, it follows from Lemma 6.12 that $\psi_{Q}(\zeta+1 / q)=\psi_{Q}(\zeta)+1 / q$. Then the region $\phi_{Q,+}\left(\Omega_{Q,+}-K(Q)\right)$ is invariant under $T_{1 / q}$, hence so is its boundary $\phi_{Q,+}(J(Q))$.

Suppose that a stretching ray $R(p / q)$ lands at some $Q \in \operatorname{Per}_{1}(1) \cap \partial \mathcal{C}(\mathbb{R})$. Let $z_{0} \in J(Q)$ be the landing point of the external ray of angle $1 / 3^{m}$ for some $m \in \mathbb{N}$. In this case we say $z_{0}$ has an external angle $1 / 3^{m}$. It is contained in the backward orbit of the parabolic fixed point $\beta_{Q}$, which has an external angle 0 . Taking $m$ sufficiently large, we may assume that $z_{0}$ is contained in the domain of $\phi_{Q,+}$. Then Lemma 6.16 implies that the point $z_{1}=\phi\left(z_{0}\right):=\phi_{Q,+}^{-1} \circ T_{1 / q} \circ \phi_{Q,+}\left(z_{0}\right)$ belongs to $J(Q)$. Here $\phi$ is a conformal map from a neighborhood $U_{0}$ of $z_{0}$, onto a round disk $B\left(z_{1}, \epsilon\right)$ centered at $z_{1}$ with radius $\epsilon$, which preserves $J(Q)$.

Lemma 6.17. $z_{1} \in J_{\text {rad }}(Q)$.

Proof. Note that translation by $1 / q$ in $\zeta_{Q}$-coordinate multiplies external angles by $3^{1 / q}$. Then, by Lemma 6.12 the external angle of $z_{1}$ is $3^{1 / q-m}$, which must be irrational. Hence $z_{1}$ is not contained in the backward orbit of $\beta_{Q}$. Now the conclusion follows from Theorem 6.15]

The following result on $J_{\text {rad }}(f)$ for a general polynomial $f$ is applicable to our case $f=Q$ and $p=z_{1}$.

Theorem 6.18. Let $f$ be a polynomial map. Then, for any point $p \in J_{\text {rad }}(f)$, there are a positive number $k>0$, a sequence of positive numbers $r_{n}>0$ tending to 0 , and a sequence of points $q_{n}$ in the basin at infinity $A_{\infty}(f)$ converging to $p$ such that

$$
B\left(q_{n}, k r_{n}\right) \subset B\left(p, r_{n}\right) \cap A_{\infty}(f) .
$$


The proof is closely related to the porosity of Julia sets for geometrically finite rational maps (cf. [P-U], $[\mathrm{Y}]$ ). The point in our case is to find a sequence of round disks converging to a point of $J(f)$ from the specified Fatou component, the basin at infinity $A_{\infty}(f)$.

The key idea is to use the following well-known Koebe distortion theorem for conformal maps (cf. [P, Theorem 1.3]).

Theorem 6.19. Let $f: B(z, r) \rightarrow \mathbb{C}$ be a conformal map. Then

(i) $B\left(f(z), \frac{1}{4} r\left|f^{\prime}(z)\right|\right) \subset f(B(z, r))$.

(ii) $\left|f^{\prime}(z)\right| \frac{1-\frac{1}{r}|w-z|}{\left(1+\frac{1}{r}|w-z|\right)^{3}} \leq\left|f^{\prime}(w)\right|$ for $w \in B(z, r)$.

Proof of Theorem 6.18. Take $p \in J_{\text {rad }}(f)$. Then there is $r>0$ satisfying that, for any $\epsilon>0$, there is a neighborhood $U$ of $p$ with $\operatorname{diam}(U)<\epsilon$ and $n>0$ such that the map $f^{n}: U \rightarrow B\left(f^{n}(p), r\right)$ is a homeomorphism. For simplicity, denote $f^{n}$ by $F$. Let $\delta>0$ be the inner radius of $U$ centered at $p$. Since $F^{-1}: B(F(p), r) \rightarrow B(p, \epsilon)$ is conformal and $B(p, \delta)$ is the inscribed disk of $U$ centered at $p$,

$$
\frac{r}{4 \delta} \leq\left|F^{\prime}(p)\right|
$$

by Theorem 6.19(i). Since $F: B(p, \delta) \rightarrow B(F(p), r)$ is conformal, from Theorem 6.19(i) and (6.1),

$$
B\left(F(p), \frac{r}{16}\right) \subset B\left(F(p), \frac{1}{4} \delta\left|F^{\prime}(p)\right|\right) \subset F(B(p, \delta)) .
$$

For $z \in J(f)$, define

$$
\begin{aligned}
h(z) & :=\sup \left\{s>0 \mid \text { there is } w \text { such that } B(w, s) \subset B\left(z, \frac{r}{16}\right) \cap A_{\infty}(f)\right\}, \\
r^{*} & :=\inf _{z \in J(f)} h(z) .
\end{aligned}
$$

We remark that $r^{*}>0$ as $h(z)$ is continuous on the compact set $J(f)$, and $r^{*}$ does not depend on $\epsilon$. From the definition of $r^{*}$, there is $q \in A_{\infty}(f)$ such that

$$
B\left(q, r^{*}\right) \subset B\left(F(p), \frac{r}{16}\right) \cap A_{\infty}(f) \subset F(B(p, \delta)) \cap A_{\infty}(f) .
$$

Since $F^{-1}: B\left(q, r^{*}\right) \rightarrow B(p, \delta)$ is conformal, by Theorem 6.19)(i),

$$
B\left(F^{-1}(q), \frac{1}{4} r^{*}\left|\left(F^{-1}\right)^{\prime}(q)\right|\right) \subset F^{-1}\left(B\left(q, r^{*}\right)\right) \subset B(p, \delta) .
$$

From Theorem 6.19(ii),

$$
B\left(F^{-1}(q), \frac{1}{4} r^{*} c\left|\left(F^{-1}\right)^{\prime}(F(p))\right|\right) \subset B\left(F^{-1}(q), \frac{1}{4} r^{*}\left|\left(F^{-1}\right)^{\prime}(q)\right|\right),
$$

where $c:=\frac{1-\frac{16}{r}\left(\frac{r}{16}-r^{*}\right)}{\left(1+\frac{16}{r}\left(\frac{r}{16}-r^{*}\right)\right)^{3}}$. Hence

$$
B\left(F^{-1}(q), \frac{1}{4} r^{*} c\left|\left(F^{-1}\right)^{\prime}(F(p))\right|\right) \subset B(p, \delta) \cap A_{\infty}(f) .
$$

On the other hand, since $F: B(p, \delta) \rightarrow B(F(p), r)$ is conformal, it follows from Theorem 6.19(i),

$$
\frac{1}{4} \delta\left|F^{\prime}(p)\right| \leq r .
$$


Hence

$$
\frac{\delta}{4 r} \leq\left|\left(F^{-1}\right)^{\prime}(F(p))\right| .
$$

Therefore combining (6.2) and (6.3), we get

$$
B\left(F^{-1}(q), \frac{c r^{*}}{16 r} \delta\right) \subset B(p, \delta) \cap A_{\infty}(f),
$$

which implies the assertion. This completes the proof of Theorem 6.18

Proof of Theorem 6.1 (rational case). The immediate basin of $\beta_{Q}$ contains two branches of the real analytic curve $\tau_{0}^{-1}(\{w=u+i v \in \mathbb{C} \mid v= \pm \sqrt{-u}\})$ for sufficiently large $-u$, terminating at $\beta_{Q}$ (cf. $[\mathrm{C}-\mathrm{G}]$ ). These curves are approximately expressed by $y= \pm C\left(x-\beta_{Q}\right)^{3 / 2}$ near $z=x+i y=\beta_{Q}$ for some $C>0$. Then, in a small neighborhood of $\beta_{Q}, A_{\infty}(Q)$ is included in the region $|y|<C\left(x-\beta_{Q}\right)^{3 / 2}$. Then, for any $k>0$, there exists $\epsilon>0$ such that the region $B\left(\beta_{Q}, \epsilon\right) \cap A_{\infty}(Q)$ cannot contain a round disk of radius $k \epsilon$. In fact, the area of the region $\{z=$ $\left.x+i y \in B\left(\beta_{Q}, \epsilon\right)|| y \mid<C\left(x-\beta_{Q}\right)^{3 / 2}\right\}$ is bounded by $\frac{4 C}{5} \epsilon^{5 / 2}$, whereas the area of the round disk is equal to $\pi(k \epsilon)^{2}$. This holds also for any point on the backward orbit of $\beta_{Q}$, which contradicts Theorem 6.18. This completes the proof of Theorem 6.1 .

Remark 6.20. Milnor pointed out that, in the family of real biquadratic polynomials $P_{a, b}(z)=\left(z^{2}+a\right)^{2}+b, a, b \in \mathbb{R}$, the stretching ray $b=a$ has Böttcher vector $1 / 2$ and lands at $(1 / 4,1 / 4)$. But this seems to be an exceptional case, because $P_{a, a}$ is the second iterate of the quadratic map $p_{a}(z)=z^{2}+a$.

\section{Accumulation sets of Stretching Rays in $\mathcal{R}_{0}$}

In this section, we will characterize the sets $I_{\eta}(\sigma)$ and $I(\eta)$ in terms of the Böttcher vector map $\eta(Q, \sigma)$. Note that the map $\eta(Q, \sigma)$ and the set $I_{\eta}(\sigma)$ depend on the choice of Fatou coordinates.

Theorem 7.1. $I_{\eta}(\sigma)=\left\{Q \in \operatorname{Per}_{1}(1) \cap \partial \mathcal{C}(\mathbb{R}) \mid \eta(Q, \sigma)=\eta\right\}$.

As a corollary, we have

Corollary 7.2. $R(\eta)$ accumulates $Q$ if and only if $\eta \in \eta(Q, \mathbb{R} / \mathbb{Z})$. Consequently, for any $Q \in \operatorname{Per}_{1}(1) \cap \partial \mathcal{C}(\mathbb{R})$, there exists at least one stretching ray accumulating $Q$.

Milnor found, in the pictures of non-landing stretching rays, that their oscillation is extremely regular and suggested the problems whether the set $I_{\eta}(\sigma)$ is a single point and whether it depends continuously on $\eta$ and $\sigma$; see Figure 2 . Considering Theorem [7.1] these are true if and only if the map $Q \mapsto \eta(Q, \sigma)$ is monotone increasing for any $\sigma$. The proof of Theorem 7.1 relies on the following.

Proposition 7.3. Fix $\sigma \in \mathbb{R} / \mathbb{Z}$ and suppose $Q_{0} \in I$, a connected component of $\operatorname{Per}_{1}(1) \cap \partial \mathcal{C}(\mathbb{R})-\tau^{-1}(\mathbb{N})$, satisfies $\eta\left(Q_{0}, \sigma\right)=\eta_{0}$. Then there exists a normalization of Fatou coordinates such that the Böttcher vector map $Q \mapsto \tilde{\eta}(Q, \sigma)$ in these new Fatou coordinates is monotone increasing on $I$ and $\tilde{\eta}\left(Q_{0}, \sigma\right)=\eta_{0}$. 
The proof of Proposition 7.3 is done by qc-deformation of the Böttcher vectors just as for the proof of Proposition [6.5 However, the conclusion is somewhat different: we have to lose the freedom of additive constant for Fatou coordinates, since Böttcher vectors depend on the choice of Fatou coordinates. In Proposition 7.3. the normalization of Fatou coordinates depends on $\sigma$. So we have not yet solved the above problems.

Proof. Suppose $\eta_{0} \in(k, k+1)$ for some $k \in \mathbb{Z}$. We regard $\sigma$ as its representative in $[0,1)$. Let $R=\varphi_{Q_{0}}\left(g_{Q_{0}, \sigma}\left(\sqrt{A\left(Q_{0}\right)}\right)\right)$ and, on the annulus $R \leq|z| \leq R^{3}$ in the Böttcher coordinate, we take a qc-map $\ell=\ell_{\eta}$ such that it changes only the radial coordinate, maps $\eta_{0}$ to $\eta \bmod \mathbb{Z}$ in the $\zeta$-coordinate for any $\eta \in(k, k+1)$ and is the identity on the boundary. We can extend it to the complement of the closed unit disk so that it commutes with $z \mapsto z^{3}$. Then $\sigma_{\eta}=\varphi_{Q_{0}}^{*} \ell^{*} \sigma_{0}$ is $Q_{0}$-invariant in the complement of $K\left(Q_{0}\right)$. Pulling it back by $g_{Q_{0}, \sigma}$, we also extend it inside $K\left(Q_{0}\right)$. Actually, it extends to the complement of $K\left(Q_{0}, \sigma\right)=J\left(Q_{0}, \sigma\right)$, the set of points which do not escape by $Q_{0}$ and $g_{Q_{0}, \sigma}$ and $J\left(Q_{0}, \sigma\right)$ has measure 0 ; see [D] or [W]. Let $\chi=\chi_{\eta}$ be the qc-map integrating $\sigma_{\eta}$ so that $Q_{\eta}=\chi \circ Q_{0} \circ \chi^{-1} \in \operatorname{Per}_{1}(1) \cap \partial \mathcal{C}(\mathbb{R})$. Its Böttcher coordinate is expressed by $\varphi_{Q_{\eta}}=\ell \circ \varphi_{Q_{0}} \circ \chi^{-1}$.

We normalize attracting Fatou coordinates by $\tilde{\phi}_{Q,-}(\sqrt{A(Q)})=0$, which is preserved under qc-deformation and repelling Fatou coordinates by $\tilde{\phi}_{Q,+}\left(\varphi_{Q}^{-1}(R)\right)=\sigma$. Then $\tilde{\phi}_{Q,+}^{-1}(\sigma)=\varphi_{Q}^{-1}(R)$ is preserved under the above qc-deformation since $R$ is fixed by $\ell$. If $\eta=\eta_{0}$, then $Q_{\eta_{0}}=Q_{0}$ and we have

$$
\phi_{Q_{0},+}\left(\varphi_{Q_{0}}^{-1}(R)\right)=\phi_{Q_{0},+}\left(g_{Q_{0}, \sigma}\left(\sqrt{A\left(Q_{0}\right)}\right)\right)=T_{\sigma}\left(\phi_{Q_{0},-}\left(\sqrt{A\left(Q_{0}\right)}\right)\right)=T_{\sigma}(0)=\sigma .
$$

Hence the original Fatou coordinate $\phi_{Q_{0},+}$ for $Q_{0}$ coincides with $\tilde{\phi}_{Q_{0},+}$.

In order to show $\tilde{\eta}\left(Q_{\eta}, \sigma\right)=\eta$, we have only to show $g:=\chi \circ g_{Q_{0}, \sigma} \circ \chi^{-1}$ is equal to the Lavaurs map $g_{Q_{\eta}, \sigma}$ of $Q_{\eta}$. Since $\sigma_{\eta}$ is, by definition, $g_{Q_{0}, \sigma}$-invariant, $g$ is holomorphic. Then $T:=\tilde{\phi}_{Q_{\eta},+} \circ g \circ \tilde{\phi}_{Q_{\eta},-}^{-1}$ is a holomorphic map from the attracting Fatou coordinate to the repelling one. We will show that it is a translation. Since

$$
\begin{aligned}
g \circ Q_{\eta} & =\chi \circ g_{Q_{0}, \sigma} \circ \chi^{-1} \circ Q_{\eta}=\chi \circ g_{Q_{0}, \sigma} \circ Q_{0} \circ \chi^{-1} \\
& =\chi \circ Q_{0} \circ g_{Q_{0}, \sigma} \circ \chi^{-1}=Q_{\eta} \circ \chi \circ g_{Q_{0}, \sigma} \circ \chi^{-1}=Q_{\eta} \circ g
\end{aligned}
$$

we have

$$
\begin{aligned}
T \circ T_{1} & =\tilde{\phi}_{Q_{\eta},+} \circ g \circ Q_{\eta} \circ \tilde{\phi}_{Q_{\eta},-}^{-1} \\
& =\tilde{\phi}_{Q_{\eta},+} \circ Q_{\eta} \circ g \circ \tilde{\phi}_{Q_{\eta},-}^{-1} \\
& =T_{1} \circ \tilde{\phi}_{Q_{\eta},+} \circ g \circ \tilde{\phi}_{Q_{\eta},-}^{-1}=T_{1} \circ T .
\end{aligned}
$$

Then $T$ induces a cylinder isomorphism from the attracting Ecalle cylinder onto the repelling one and hence is a translation.

Next we show $T=T_{\sigma}$. The critical point $\sqrt{A}$ is preserved by qc-map $\chi$ and takes 0 in the attracting Fatou coordinate. On the other hand, the value $\tilde{\phi}_{Q,+}^{-1}(\sigma)$ is preserved by $\chi$. When $\eta=\eta_{0}, T$ is equal to $T_{\sigma}$ and maps $0=\tilde{\phi}_{Q_{0},-}\left(\sqrt{A\left(Q_{0}\right)}\right)$ to $\sigma=\tilde{\phi}_{Q_{0},+}\left(\varphi_{Q_{0}}^{-1}(R)\right)$. Hence, this is true for any $\eta$ and we also have $T=T_{\sigma}$ for any $\eta$. Thus $g=g_{Q_{\eta}, \sigma}$. This completes the proof.

Proof of Theorem 7.1. Considering Lemma6.10 we have only to show $\left\{Q \in \operatorname{Per}_{1}(1)\right.$ $\left.\cap \partial \mathcal{C}(\mathbb{R}) \mid \eta(Q, \sigma)=\eta_{0}\right\} \subset I_{\eta_{0}}(\sigma)$. Suppose $\eta\left(Q_{0}, \sigma\right)=\eta_{0}$. We apply Proposition 
7.3. Then the Böttcher vector map $Q \mapsto \tilde{\eta}(Q, \sigma)$ for the new Fatou coordinates is monotone increasing. Let $P_{n}$ be a sequence on $R\left(\eta_{0}\right)$ satisfying $P_{n} \rightarrow Q_{1}$ and $\sigma\left(P_{n}\right) \rightarrow \sigma$ with respect to the new Fatou coordinates. Then $\tilde{\eta}\left(Q_{1}, \sigma\right)=\eta_{0}$. Since $Q \mapsto \tilde{\eta}(Q, \sigma)$ is monotone, this implies $Q_{1}=Q_{0}$ and we have $\tilde{I}_{\eta_{0}}(\sigma)=\left\{Q_{0}\right\}$. Since the repelling Fatou coordinates coincide at $Q_{0}$ in both normalizations, it follows that, by Lemma 7.4, $Q_{0} \in I_{\eta_{0}}(\sigma)$ in the original normalization. This completes the proof of Theorem 7.1

Take two Fatou coordinates $\phi_{P, \pm}^{1}$ and $\phi_{P, \pm}^{2}$ for $P$. They differ only by constants: $\phi_{P, \pm}^{2}(z)-\phi_{P, \pm}^{1}(z)=\delta_{P, \pm}$ depending on $P$. Hence their lifted phases satisfy

$$
\tilde{\sigma}_{2}(P)=\phi_{P,+}^{2}(z)-\phi_{P,-}^{2}(z)=\tilde{\sigma}_{1}(P)+\delta_{P,+}-\delta_{P,-} .
$$

And, if $P_{n} \rightarrow Q$ and $\sigma_{1}\left(P_{n}\right) \rightarrow \sigma_{1}$, we have

$$
\sigma_{2}\left(P_{n}\right)=\sigma_{1}\left(P_{n}\right)+\delta_{P_{n},+}-\delta_{P_{n},-} \rightarrow \sigma_{2}:=\sigma_{1}+\delta_{Q,+}-\delta_{Q,-} \quad(\bmod \mathbb{Z}) .
$$

Lemma 7.4. Denote the corresponding impressions by $I_{\eta}^{1}\left(\sigma_{1}\right)$ and $I_{\eta}^{2}\left(\sigma_{2}\right)$. Then $Q \in I_{\eta}^{1}\left(\sigma_{1}\right)$ if and only if $Q \in I_{\eta}^{2}\left(\sigma_{2}\right)$. Especially, if $\delta_{Q, \pm}=0$, then $\sigma_{2}=\sigma_{1}$ and $Q \in I_{\eta}^{2}\left(\sigma_{1}\right)$ if and only if $Q \in I_{\eta}^{1}\left(\sigma_{1}\right)$.

Remark 7.5. This lemma does not insist that $I_{\eta}^{1}\left(\sigma_{1}\right)=I_{\eta}^{2}\left(\sigma_{2}\right)$, because $\sigma_{1}$ and $\sigma_{2}$ depend on $Q$.

\section{REFERENCES}

[B] B. Branner: Turning around the connectedness locus. In: "Topological methods in modern Mathematics." pp. 391-427. Houston, Publish or Perish, 1993. MR 94c:58168

[B-D] B. Branner and A. Douady: Surgery on complex polynomials. In: "Holomorphic Dynamics." Lect. Notes in Math. 1345, pp. 11-72, 1988. MR 90e:58114

[B-H1] B. Branner and J. Hubbard: The iteration of cubic polynomials. Part I: The global topology of parameter space. Acta Math. 160, 143-206 (1988). MR 90d:30073

[B-H2] : The iteration of cubic polynomials. Part II: Patterns and parapatterns. Acta Math. 169, 229-325 (1992). MR 94d:30044

[Bu-He] X. Buff and C. Henriksen: Julia sets in parameter spaces. Comm. Math. Phys. 220, 333-375 (2001). MR 2002d:37075

[C-G] L. Carleson and T. Gamelin: Complex Dynamics. Springer-Verlag, 1993. MR 94h:30033

[D] A. Douady: Does a Julia set depend continuously on the polynomials? Proceedings of Symposia in Applied Mathematics 49, pp. 91-138, 1994.

[D-H] A. Douady and J. Hubbard: On the dynamics of polynomial-like mappings. Ann. Sci. Ec. Norm. Sup. 18, 287-343 (1985). MR 87f:58083

[E-Y] A. Epstein and M. Yampolsky: Geography of the cubic connectedness locus I: Intertwining surgery. Ann. Sci. Ec. Norm. Sup. 32, 151-185 (1999). MR 2000i:37067

[G-S] J. Graczyk and G. Świątek: Generic hyperbolicity in the logistic family. Annals Math. 146, 1-52 (1997). MR 99b:58079

[K] J. Kiwi: Rational rays and critical portraits of complex polynomials. Stony Brook IMS Preprint 1997/15, 1997.

[La] P. Lavaurs: Systèmes dynamiques holomorphes: explosion de points périodiques paraboliques. These Univ. Paris-Sud, 1989.

[Ly] M. Lyubich: Dynamics of quadratic polynomials, I-II. Acta Math. 178, 185-297 (1997). MR 98e:58145

[Mc] C. McMullen: Hausdorff dimension and conformal dynamics II: Geometrically finite rational maps. Comment. Math. Helv. 75, 535-593 (2000). MR 2001m:37089

[Mi1] J. Milnor: Remarks on iterated cubic maps. Experimental Math. 1, 5-23 (1992). MR 94c:58096

[Mi2] : Dynamics in one complex variable: Introductory Lectures. Vieweg (1999). MR 2002i:37057 
[P] C. Pommerenke: Boundary Behaviour of Conformal Maps. Springer-Verlag, 1991. MR 95b:30008

[P-U] F. Przytycki and M. Urbański: Porosity of Julia sets of non-recurrent and parabolic Collet-Eckmann rational functions. Ann. Acad. Sci. Fenn. Math. 26, 125-154 (2001). MR 2002b:37063

[Sh] M. Shishikura: The Hausdorff dimension of the boundary of the Mandelbrot set and Julia sets. Annals Math. 147, 225-267 (1998). MR 2000f:37056

[W] P. Willumsen: Holomorphic Dynamics: On accumulation of stretching rays. Ph.D. thesis Tech. Univ. Denmark, 1997.

[Y] Y. Yin: Geometry and dimension of Julia sets. In: "Tan Lei (eds.) The Mandelbrot set, Theme and Variations." Lecture Notes Series 274, pp. 281-287. London Math. Soc., 2000. MR 2001d:37062

Department of Mathematics, Osaka City University, Sugimoto 3-3-138, Sumiyoshi-ku, OSAKA 558-8585, JAPAN

E-mail address: komori@sci.osaka-cu.ac.jp

Tokyo Polytechnic University, 1583 Iiyama, Atsugi, Kanagawa 243-0297, Japan

E-mail address: nakane@gen.t-kougei.ac.jp 